Homology, Homotopy and Applications, vol. 20(1), 2018, pp.87-116

\title{
RELATIVE HOMOLOGICAL ALGEBRA AND WALDHAUSEN $K$-THEORY
}

\author{
ANDREW SALCH \\ (communicated by Charles A. Weibel)
}

\begin{abstract}
We study the question of the existence of a Waldhausen category on any (relative) abelian category in which the contractible objects are the (relatively) projective objects. The associated $K$-theory groups are "stable algebraic $G$-theory," which in degree zero form a certain stable representation group. We prove both some existence and nonexistence results about such Waldhausen category structures, including the fact that, while it was known that the category of $R$-modules admits a model category structure if $R$ is quasi-Frobenius, that assumption is required even to get a Waldhausen category structure with cylinder functor-i.e., Waldhausen categories do not offer a more general framework than model categories for studying stable representation theory of rings. We study multiplicative structures on these Waldhausen categories, and we relate stable algebraic $G$-theory to algebraic $K$-theory and we compute stable algebraic $G$-theory for finite-dimensional quasi-Frobenius nilpotent extensions of finite fields. Finally, we show that the connective stable $G$-theory spectrum of $\mathbb{F}_{p^{n}}[x] / x^{p^{n}}$ is a complex-orientable ring spectrum, partially answering a question of J. Morava about complex orientations on algebraic $K$-theory spectra.
\end{abstract}

\section{Introduction}

Waldhausen's paper [14] defines several kinds of categorical structure which are meaningful for algebraic $K$-theory. A category with cofibrations and weak equivalences, also called a Waldhausen category, has just enough structure for Waldhausen's machinery to produce an associated $K$-theory infinite loop space. A Waldhausen category which satisfies additional axioms and/or has additional structure will have better properties which, e.g., make the problem of actually computing the associated $K$-theory more tractable. For example, a Waldhausen category satisfying the "extension axiom" and the "saturation axiom" and equipped with an additional structure called a "cylinder functor" admits Waldhausen's Localization Theorem (see [14]),

Received November 4, 2015, revised June 29, 2017; published on January 3, 2018.

2010 Mathematics Subject Classification: 18G25, 13C60, 19 D55.

Key words and phrases: Waldhausen category, algebraic $K$-theory, quasi-Frobenius ring, relative homological algebra, complex oriented ring spectrum.

Article available at http://dx.doi.org/10.4310/HHA.2018.v20.n1.a7

Copyright (C) 2018, International Press. Permission to copy for private use granted. 
a computationally powerful result that describes the sense in which localizations of the Waldhausen category induce long exact sequences in the $K$-theory groups.

Meanwhile, in stable representation theory, one regards the projective modules over a ring as "contractible," and maps of modules that factor through projective modules are regarded as "nulhomotopic." This suggests that the category of modules over a ring perhaps has a Waldhausen category structure in which the objects weakly equivalent to zero - the contractible objects - are precisely the projective modules. More generally, one has the tools of relative homological algebra: if one chooses a sufficiently well-behaved class of objects in an abelian category, one can do a form of homological algebra in which the chosen class of objects plays the role of projective objects. Given an abelian category and a class of relative projective objects, one wants to know if there is a natural Waldhausen category structure on that abelian category, such that the contractible objects are precisely the relative projectives. One also wants to know how many extra axioms are satisfied by, and how much additional structure is admitted by, such a Waldhausen category.

In this paper we prove the following theorems that answer the above questions, and explain fundamental properties of the relationships between Waldhausen $K$-theory, relative homological algebra, and stable representation theory:

1. Definition-Proposition 2.19: Given an abelian category $\mathcal{C}$ and a sufficiently nice pair of allowable classes $E, F$ in $\mathcal{C}$, there exists a Waldhausen category structure on $\mathcal{C}$ whose weak equivalences are the $E$-stable equivalences and whose cofibrations are the $F$-monomorphisms. In particular, the $E$-projective objects are precisely the contractible objects in this Waldhausen category. This Waldhausen category satisfies the saturation axiom and the extension axiom.

2. Theorem 3.4: $\mathcal{C}$ has a cylinder functor satisfying the cylinder axiom if and only if $\mathcal{C}$ obeys a certain generalized quasi-Frobenius condition: every object must functorially embed in an $E$-projective object by an $F$-monomorphism.

3. As a consequence, we have Corollary 3.7: any quasi-Frobenius abelian category with enough projectives and functorially enough injectives admits the structure of a Waldhausen category whose weak equivalences are the stable equivalences and whose cofibrations are the monomorphisms. This Waldhausen category has a cylinder functor, and it satisfies the saturation, extension, and cylinder axioms.

4. As a consequence, we have Corollary 3.8: if $R$ is a finite-dimensional quasiFrobenius algebra over a finite field, then the category of finitely generated (left) $R$-modules admits the structure of a Waldhausen category in which the cofibrations are the monomorphisms and the weak equivalences are the stable equivalences. (A similar result follows from well-known results in model category theory, if one instead considers the category of all $R$-modules, not only finitelygenerated $R$-modules; but the usual tools for building the model structure, especially Quillen's small object argument, are not applicable to the category of finitely generated modules. See [6].) This Waldhausen category admits a cylinder functor and satisfies the saturation, extension, and cylinder axioms.

5. In Proposition 3.12 we show that, for a finite-dimensional co-commutative Hopf algebra over a finite field, this Waldhausen category is even better: it has a multiplicative structure coming from the tensor product of modules, and this 
multiplicative structure gives rise to the structure of a homotopy-commutative ring spectrum on the $K$-theory spectrum of this Waldhausen category.

6 . In the appendix, section 5 , we prove a theorem to the effect that, under appropriate set-theoretic conditions on $\mathcal{C}$, if every object embeds in an $E$-projective object by an $F$-monomorphism, then such embeddings can be chosen functorially. This is Theorem 5.5. So the word "functorially" can, under some reasonable hypotheses on $\mathcal{C}$, be omitted from the above statements.

7. We use the theorems described above to prove our Proposition 4.4, in which we show that, for finite-dimensional quasi-Frobenius nilpotent extensions of finite fields, stable $G$-theory is a delooping of relative algebraic $K$-theory.

8. As a consequence, we get our Theorem 4.6, in which we prove that, under the same assumptions on the ring, stable $G$-theory in positive degrees vanishes $\ell$ adically for $\ell \neq p$, and is isomorphic to topological cyclic homology with a degree shift $p$-adically, where $p$ is the characteristic of the base field.

9. Finally, in Theorem 4.8, we compute the stable $G$-theory groups $\left(G_{s t}\right)_{*}\left(\mathbb{F}_{p}[x] / x^{p^{n}}\right)$ of truncated polynomial algebras over $\mathbb{F}_{p}$, in terms of the Hesselholt-Madsen description of the topological cyclic homology of truncated polynomial algebras. We provide a partial answer to a question (unpublished) of J. Morava: under what circumstances does an algebraic $K$-theory ring spectrum admit a complex orientation? ${ }^{1}$ Our answer is that the stable $G$-theory spectrum $G_{s t}\left(\mathbb{F}_{p}[x] / x^{p^{n}}\right)$ is, for all $p$ and $n$, a complex-orientable ring spectrum.

"Quasi-Frobenius conditions" appear prominently throughout this paper. Recall that a ring $R$ is said to be quasi-Frobenius if every projective $R$-module is injective and vice versa. The appearance of these conditions in connection with Waldhausen $K$-theory stems from the theorem of Faith and Walker (see [4]):

Theorem 1.1. (Faith-Walker) $A$ ring $R$ is quasi-Frobenius if and only if every $R$-module embeds in a projective $R$-module.

Here is one point of view on the significance of Theorem 3.4 in the present paper. It has been known for a long time, e.g., as described in [6], that when $R$ is a quasiFrobenius ring, there exists a model category structure on the category of $R$-modules in which the cofibrations are the injections and the weak equivalences are the stable equivalences of modules. Constructing this model category structure uses the quasiFrobenius condition in an essential way. But a Waldhausen category structure on $R$-modules is weaker, less highly-structured, than a model category structure; so one might hope that, even in the absence of the quasi-Frobenius condition on $R$, one could put the structure of a Waldhausen category on the category of $R$-modules, such that the cofibrations are the injections and the weak equivalences are the stable equivalences of modules. As a consequence of Theorem 3.4, one only gets a Waldhausen category structure with cylinder functor on the category of $R$-modules if $R$ is

\footnotetext{
${ }^{1}$ While Waldhausen's $w S_{\bullet}$-construction produces an infinite loop space, it is much more standard to speak of complex orientations on a (ring) spectrum, rather than on an infinite loop space. We will use, whenever convenient and without comment, the classical equivalence between connective spectra and infinite loop spaces; see [10]. Under this equivalence, the (unstable) homotopy groups of the infinite loop space agree with the (stable) homotopy groups of its associated spectrum.
} 
quasi-Frobenius. So the category of R-modules admits a model category structure as desired if and only if it admits a Waldhausen category structure with cylinder functor as desired. (But our results, such as Corollary 3.7 on existence of the cylindrical Waldhausen category structure, also have the virtue of applying to quasi-Frobenius abelian categories that are not categories of modules over a ring.)

So one knows that, when $R$ is a quasi-Frobenius ring, then one has the model category of $R$-modules with cofibrations inclusions and weak equivalences the stable equivalences, and from the theorems in this paper which we have described above, one knows that relaxing the condition that $R$ be quasi-Frobenius does not enable one to get cylindrical Waldhausen category structures in any greater generality. Then one asks the natural question: restricting to the finitely-generated $R$-modules, what are the K-groups of this Waldhausen category? That leads us to the computations of Theorem 4.6 and Theorem 4.8.

Our stable $G$-theory Waldhausen category poses an alternative to a construction by Garkusha in [5], who constructs a Waldhausen category which models the cofiber (on the spectrum level) of the Cartan map from $K$-theory to $G$-theory. Garkusha's construction is a Waldhausen category structure on chain complexes of $R$-modules, and when $R$ is finite and quasi-Frobenius, our Proposition 4.3 also describes the cofiber of the Cartan map but with a much smaller model than Garkusha's (the stable $G$-theory Waldhausen category structure on $R$-modules, rather than on chain complexes of $R$-modules). Our stable $G$-theory, as a model for the cofiber of the Cartan map, also has the advantage of multiplicative structure, as in Proposition 3.12.

\section{Acknowledgments}

We would not have written this paper or thought about any of these issues if not for conversations we had with C. Ogle, who taught us a great deal about Waldhausen $K$-theory during the summer of 2012. We are grateful to C. Ogle for his generosity in teaching us about this subject. We also thank the anonymous referee for their helpful and useful comments, and C. Weibel for his editorial help.

\section{Waldhausen category structures from allowable classes on abelian categories}

\subsection{Definitions}

This subsection is entirely review and there are no new results or definitions in it, with the exception of Definition 2.8 and Definition-Proposition 2.10.

Throughout this subsection, let $\mathcal{C}$ be an abelian category.

We begin with the definition of an allowable class, which is the structure one needs to specify on $\mathcal{C}$ in order to have a notion of relative homological algebra in $\mathcal{C}$.

Definition 2.1. An allowable class in $\mathcal{C}$ consists of a collection $E$ of short exact sequences in $\mathcal{C}$ which is closed under isomorphism of short exact sequences and which contains every short exact sequence in which at least one object is the zero object of $\mathcal{C}$. (See section IX.4 of [7] for this definition and basic properties.)

The usual "absolute" homological algebra in an abelian category $\mathcal{C}$ is recovered by letting the allowable class $E$ consist of all short exact sequences in $\mathcal{C}$. 
Once one chooses an allowable class $E$, one has the notion of monomorphisms relative to $E$, or " $E$-monomorphisms," and epimorphisms relative to $E$, or " $E$-epimorphisms."

Definition 2.2. Let $E$ be an allowable class in $\mathcal{C}$. A monomorphism $f: M \rightarrow N$ in $\mathcal{C}$ is called an E-monomorphism or an E-monic if the short exact sequence $0 \rightarrow M \stackrel{f}{\longrightarrow}$ $N \rightarrow$ coker $f \rightarrow 0$ is in $E$.

Dually, an epimorphism $g: M \rightarrow N$ is called an E-epimorphism or an E-epic if the short exact sequence $0 \rightarrow \operatorname{ker} f \rightarrow M \stackrel{f}{\longrightarrow} N \rightarrow 0$ is in $E$.

When $E$ consists of all short exact sequences in $\mathcal{C}$, the $E$-monomorphisms are simply the monomorphisms, and the $E$-epimorphisms are simply the epimorphisms.

In relative homological algebra, one has the notion of relative projectives, or $E$ projectives: these are simply the objects which lift over every $E$-epimorphism. The $E$-injectives are defined dually.

Definition 2.3. Let $E$ be an allowable class in $\mathcal{C}$. An object $X$ of $\mathcal{C}$ is said to be an E-projective if, for every diagram with $f E$-epic,

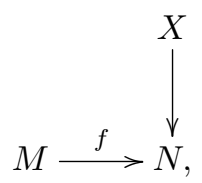

there exists a morphism $X \rightarrow M$ making the above diagram commute.

Dually, $X$ is said to be an E-injective if, for every diagram with $f E$-monic,

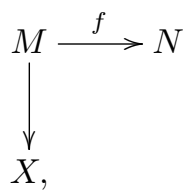

there exists a morphism $N \rightarrow X$ making the above diagram commute.

When the allowable class $E$ is clear from context we sometimes refer to $E$-projectives and $E$-injectives as relative projectives and relative injectives, respectively.

In the absolute case, the case that $E$ is all short exact sequences in $\mathcal{C}$, the $E$ projectives are simply the projectives, and the $E$-injectives are simply the injectives.

Once one has a notion of relative projectives, one has a notion of a stable equivalence, i.e., a "homotopy" between maps, as studied in stable representation theory:

Definition 2.4. Let $E$ be an allowable class in $\mathcal{C}$. Let $f, g: M \rightarrow N$ be morphisms in $\mathcal{C}$. We say that $f$ and $g$ are $E$-stably equivalent and we write $f \simeq g$ if $f-g$ factors through an $E$-projective object of $\mathcal{C}$.

One can then define stable equivalence of objects, i.e., "homotopy equivalence":

Definition 2.5. We say that a map $f: M \rightarrow N$ is a E-stable equivalence if there exists a map $h: N \rightarrow M$ such that $f \circ h \simeq \operatorname{id}_{N}$ and $h \circ f \simeq \operatorname{id}_{M}$. 
In the absolute case where $E$ consists of all short exact sequences in $\mathcal{C}$, this is the usual notion of stable equivalence of modules over a ring. Over a quasi-Frobenius ring, stably equivalent modules have isomorphic cohomology in positive degrees, so if one is serious about computing the cohomology of all finitely-generated modules over a particular Hopf algebra, it is natural to first compute the representation ring modulo stable equivalence. See [9] for this useful perspective (which motivates much of the work in this paper).

We now define the relative-homological-algebraic generalizations of an abelian category having enough projectives or enough injectives. We provide an extra twist on these definitions as well, which we will need for certain theorems: sometimes we will need to know that, for example, not only does every object embed in an injective, but that we can choose such embeddings in a functorial way.

Definition 2.6. Let $E$ be an allowable class in $\mathcal{C}$. We say that $\mathcal{C}$ has enough $E$ projectives if, for any object $M$ of $\mathcal{C}$, there exists an $E$-epic $N \rightarrow M$ with $N$ an $E$-projective. We say that $\mathcal{C}$ has functorially enough $E$-projectives if $\mathcal{C}$ has enough $E$ projectives and the choice of $E$-epimorphisms from $E$-projectives to each object of $\mathcal{C}$ can be made functorially, i.e., there exists a functor $P: \mathcal{C} \rightarrow \mathcal{C}$ together with a natural transformation $\epsilon: P \rightarrow \operatorname{id}_{\mathcal{C}}$ such that $P(X)$ is $E$-projective and $\epsilon(X): P(X) \rightarrow X$ is an $E$-epimorphism for all objects $X$ of $\mathcal{C}$, and such that, if $f: X \rightarrow Y$ is an $E$ epimorphism, then so is $P(f): P(X) \rightarrow P(Y)$.

Dually, we say that $\mathcal{C}$ has enough $E$-injectives if, for any object $M$ of $\mathcal{C}$, there exists an $E$-monic $M \rightarrow N$ with $N$ an $E$-injective. We say that $\mathcal{C}$ has functorially enough $E$-injectives if $\mathcal{C}$ has enough $E$-injectives and the choice of $E$-monomorphisms into $E$-injectives from each object of $\mathcal{C}$ can be made functorially, i.e., there exists a functor $I: \mathcal{C} \rightarrow \mathcal{C}$ together with a natural transformation $\eta: \operatorname{id}_{\mathcal{C}} \rightarrow I$ such that $I(X)$ is $E$ injective and $\eta(X): X \rightarrow I(X)$ is an $E$-monomorphism for all objects $X$ of $\mathcal{C}$, and such that, if $f: X \rightarrow Y$ is an $E$-monomorphism, then so is $I(f): I(X) \rightarrow I(Y)$.

Our need to have abelian categories with functorially enough injectives or projectives is only due to Waldhausen's definitions of cylinder functors and resulting theorems, in [14], demanding that cylinder functors actually be functors. In section 5 we show that, under appropriate set-theoretic hypotheses on $\mathcal{C}$, having enough injectives implies having functorially enough injectives.

Finally, we have our first definition of a quasi-Frobenius condition:

Definition 2.7. Let $E$ be an allowable class in $\mathcal{C}$. We will call $E$ a quasi-Frobenius allowable class if the following conditions are both satisfied:

- $\mathcal{C}$ has enough $E$-projectives and enough $E$-injectives, and

- the $E$-projectives are exactly the $E$-injectives.

If the allowable class consisting of all short exact sequences in $\mathcal{C}$ is a quasi-Frobenius class, then we will simply say that $\mathcal{C}$ is quasi-Frobenius.

Here are some important examples of allowable classes in abelian categories:

- As described above, the usual "absolute" homological algebra in an abelian category $\mathcal{C}$ is recovered by letting the allowable class $E$ consist of all short exact sequences in $\mathcal{C}$; then the $E$-projectives are the usual projectives, etc. Note 
that, if $E$ is an arbitrary allowable class in $\mathcal{C}$, then any projective object is an $E$-projective object, but the converse is not necessarily true.

- Suppose $\mathcal{C}, \mathcal{D}$ are abelian categories and $F: \mathcal{C} \rightarrow \mathcal{D}$ is an additive functor. Then we can let $E$ be the allowable class in $\mathcal{C}$ consisting of the short exact sequences which are sent by $F$ to split short exact sequences in $\mathcal{D}$. If $F$ has a left (resp. right) adjoint $G$ then objects of $\mathcal{C}$ of the form $G F X$ (resp. $F G X$ ) are $E$ projectives (resp. $E$-injectives) and the counit map $G F X \rightarrow X$ of the comonad $G F$ (resp. the unit map $X \rightarrow G F X$ of the monad $G F$ ) is an $E$-epic (resp. $E$ monic), hence $\mathcal{C}$ has enough $E$-projectives (resp. enough $E$-injectives). These ideas are in $[\mathbf{7}]$.

For example, if $R$ is a ring and $\mathcal{C}$ the category of $R$-modules and $\mathcal{D}$ the category of abelian groups, and $F$ the forgetful functor, then $E$ is the class of short exact sequences of $R$-modules whose underlying short exact sequences of abelian groups are split. The $R$-modules of the form $R \otimes_{\mathbb{Z}} M$, for $M$ an $R$-module, are $E$-projectives.

Here is a definition which the author introduced in [13], and which makes many arguments involving allowable classes substantially smoother:

Definition 2.8. An allowable class $E$ is said to have retractile monics if, whenever $g \circ f$ is an $E$-monic, $f$ is also an $E$-monic.

Dually, an allowable class $E$ is said to have sectile epics if, whenever $g \circ f$ is an $E$-epic, $g$ is also an $E$-epic.

The utility of the notion of "having sectile epics" comes from the following fundamental theorem of relative homological algebra, due to Heller (see [7]), whose statement is slightly cleaner if one is willing to use the phrase "having sectile epics." The consequence of Heller's theorem is that, in order to specify a "reasonable" allowable class in an abelian category, it suffices to specify the relative projective objects associated to it.

Theorem 2.9. If $\mathcal{C}$ is an abelian category and $E$ is an allowable class in $\mathcal{C}$ with sectile epics and enough E-projectives, then an epimorphism $M \rightarrow N$ in $\mathcal{C}$ is an $E$ epic if and only if the induced map $\operatorname{hom}_{\mathcal{C}}(P, M) \rightarrow \operatorname{hom}_{\mathcal{C}}(P, N)$ of abelian groups is an epimorphism for all E-projectives $P$.

Heller's theorem suggests the following construction: if $E$ is an allowable class, we can construct a "sectile closure of $E$ " which has the same relative projectives and the same stable equivalences but which has sectile epics. Here are the specific properties of this construction (we have neglected to write out proofs of these properties because the proofs are so elementary):

Definition-Proposition 2.10. Let $\mathcal{C}$ be an abelian category, $E$ an allowable class in $\mathcal{C}$. Let $E_{\text {sc }}$ be the allowable class in $\mathcal{C}$ consisting of the exact sequences $X \rightarrow Y \rightarrow Y / X$ such that the induced map $\operatorname{hom}_{\mathcal{C}}(P, Y) \rightarrow \operatorname{hom}_{\mathcal{C}}(P, Y / X)$ is a surjection of abelian groups for every E-projective $P$. We call $E_{s c}$ the sectile closure of $E$. The allowable class $E_{\text {sc }}$ has the following properties:

- $E_{s c}$ has sectile epics.

- An object of $\mathcal{C}$ is an E-projective if and only if it is an $E_{s c}$-projective. 
- If $f, g$ are two morphisms in $\mathcal{C}$ then $f$ and $g$ are E-stably equivalent if and only if they are $E_{s c}$-stably equivalent.

- If $X, Y$ are two objects in $\mathcal{C}$ then $X$ and $Y$ are E-stably equivalent if and only if they are $E_{s c}$-stably equivalent.

- $\left(E_{s c}\right)_{s c}=E_{s c}$.

- If $E, F$ are two allowable classes in $\mathcal{C}$ and $F \subseteq E$ then $F_{s c} \subseteq E_{s c}$.

Of course there is a construction dual to the sectile closure, a retractile closure, with dual properties, but with a less straightforward relationship to stable equivalence, since stable equivalence is defined in terms of projectives, not injectives.

We now recall Waldhausen's definitions:

Definition 2.11. A pointed category $\mathcal{C}$, with pushouts, equipped with a specified class of cofibrations and a specified class of weak equivalences, both closed under composition, is called a Waldhausen category if the following axioms are satisfied:

- (Cof 1) The isomorphisms in $\mathcal{C}$ are cofibrations.

- (Cof 2) For every object $X$ of $\mathcal{C}$, the map pt $\rightarrow \mathcal{C}$ is a cofibration. (We write pt for the zero object of $\mathcal{C}$.)

- (Cof 3) If $X \rightarrow Y$ is a morphism in $\mathcal{C}$ and $X \rightarrow Z$ is a cofibration, then the canonical map $Y \rightarrow Y \coprod_{X} Z$ is a cofibration.

- (Weq 1) The isomorphisms in $\mathcal{C}$ are weak equivalences.

- (Weq 2) If

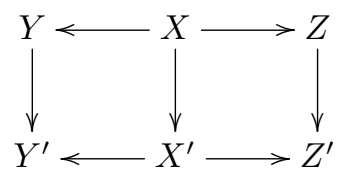

is a commutative diagram in $\mathcal{C}$ in which the maps $X \rightarrow Y$ and $X^{\prime} \rightarrow Y^{\prime}$ are cofibrations and all three vertical maps are weak equivalences, then the induced map $Y \coprod_{X} Z \rightarrow Y^{\prime} \coprod_{X^{\prime}} Z^{\prime}$ is a weak equivalence.

Ultimately, if $\mathcal{C}$ is a Waldhausen category, then what one typically wants to understand is $|w S . \mathcal{C}|$, the geometric realization of the simplicial category $w S . \mathcal{C}$ constructed by Waldhausen in [14]. The $K$-groups of $\mathcal{C}$ are defined as the homotopy groups of the loop space $\Omega|w S . \mathcal{C}|$, that is, $\pi_{n+1}(|w S . \mathcal{C}|) \cong \pi_{n}(\Omega|w S . \mathcal{C}|) \cong K_{n}(\mathcal{C})$.

If $\mathcal{C}$ is a Waldhausen category then the following axioms may or may not be satisfied:

Definition 2.12. - (Saturation axiom) If $f, g$ are composable maps in $\mathcal{C}$ and two of $f, g, g \circ f$ are weak equivalences then so is the third.

- (Extension axiom) If

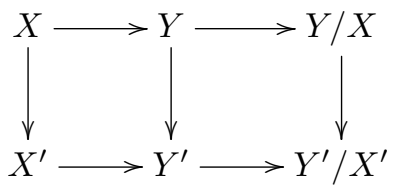

is a map of cofiber sequences and the maps $X \rightarrow X^{\prime}$ and $Y / X \rightarrow Y^{\prime} / X^{\prime}$ are weak equivalences then so is the map $Y \rightarrow Y^{\prime}$. 
The following definition of a cylinder functor, Definition 2.13, is Definition IV.8.8 of [15]. It is somewhat weaker than Waldhausen's original definition, from [14], but is still strong enough to prove Waldhausen's Fibration Theorem (reproduced below as Theorem 2.14).

Definition 2.13. If $\mathcal{C}$ is a Waldhausen category, a cylinder functor on $\mathcal{C}$ is a functor from the category of arrows $f: X \rightarrow Y$ in $\mathcal{C}$ to the category of diagrams of the form

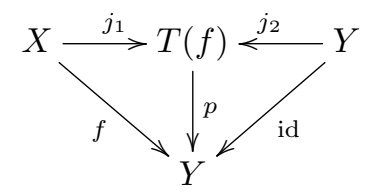

in $\mathcal{C}$ satisfying the three conditions:

- (Cyl 1) If

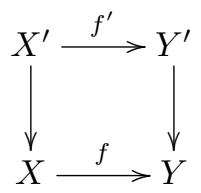

is a commutative diagram in $\mathcal{C}$ in which the vertical maps are weak equivalences (resp. cofibrations), then the map $T\left(f^{\prime}\right) \rightarrow T(f)$ is a weak equivalence (resp. cofibration and

$$
X \coprod_{X^{\prime}} T\left(f^{\prime}\right) \coprod_{Y^{\prime}} Y \rightarrow T(f)
$$

is a cofibration).

- (Cyl 2) $T($ pt $\rightarrow Y)=Y$ and the maps $p$ and $j_{2}$ are the identity map on $Y$.

- (Cyl 3) The map $j_{1} \coprod j_{2}: X \coprod Y \rightarrow T(f)$ is a cofibration.

A Waldhausen category with cylinder functor may or may not satisfy the additional condition:

- (Cylinder axiom) For any map $f$ in $\mathcal{C}$, the map $p$ is a weak equivalence.

The idea here is that a cylinder functor satisfying the cylinder axiom acts very much like the mapping cylinder construction from classical homotopy theory - or, more generally, like the cofibration-acyclic-fibration factorization system in a model category. Some of Waldhausen's most powerful results in [14] have proofs of a sufficiently homotopy-theoretic flavor that they require that every Waldhausen category in sight has a cylinder functor obeying the cylinder axioms. A good example of this is Waldhausen's Fibration Theorem, which we now recall (see V.2.1 of [15] for a proof using the version of cylinder functors defined in Definition 2.13):

Theorem 2.14. Fibration Theorem (Waldhausen). Suppose $\mathcal{C}, \mathcal{C}_{0}$ are Waldhausen categories with the same underlying category and the same underlying class of cofibrations. Suppose all of the following conditions are satisfied:

- Every weak equivalence in $\mathcal{C}$ is also a weak equivalence in $\mathcal{C}_{0}$.

- $\mathcal{C}_{0}$ admits a cylinder functor satisfying the cylinder axiom.

- The weak equivalences in $\mathcal{C}_{0}$ satisfy the saturation and extension axioms. 
Then $|w S . \mathcal{X}| \rightarrow|w S . \mathcal{C}| \rightarrow\left|w S . \mathcal{C}_{0}\right|$ is a homotopy fibre sequence, where $\mathcal{X}$ is the full sub-Waldhausen-category of $\mathcal{C}$ generated by the objects that are weakly equivalent to pt in $\mathcal{C}_{0}$. As a consequence, after looping and taking homotopy groups, we get the long exact sequence of $K$-groups:

$$
\cdots \rightarrow K_{n+1}\left(\mathcal{C}_{0}\right) \rightarrow K_{n}(\mathcal{X}) \rightarrow K_{n}(\mathcal{C}) \rightarrow K_{n}\left(\mathcal{C}_{0}\right) \rightarrow K_{n-1}(\mathcal{X}) \rightarrow \cdots
$$

The question of when our Waldhausen categories given by allowable classes on abelian categories satisfy the required conditions for the Fibration Theorem to hold is the subject of most of this paper.

\subsection{The Waldhausen category structure on an abelian category associated to a pair of allowable classes}

In this subsection, we will prove that, under appropriate hypotheses, an abelian category equipped with a pair of allowable classes $E, F$ admits a Waldhausen category structure in which the cofibrations are the $F$-monomorphisms and the weak equivalences are the $E$-stable equivalences. In this subsection we will also find conditions under which this Waldhausen category satisfies the extension and saturation axioms (see Definition 2.12 for definitions of these axioms). To get anywhere, we will need some lemmas:

Lemma 2.15. A pullback of a surjective map of abelian groups is surjective.

Proof. Elementary.

Lemma 2.16. Let $\mathcal{C}$ be an abelian category and let $E$ be an allowable class with retractile monics. Suppose $\mathcal{C}$ has enough E-injectives. Then E-monics are closed under pushout in $\mathcal{C}$. That is, if $X \rightarrow Z$ is an $E$-monic and $X \rightarrow Y$ is any morphism in $\mathcal{C}$, then the canonical map $Y \rightarrow Y \coprod_{X} Z$ is an E-monic.

Proof. Suppose $f: X \rightarrow Z$ is an $E$-monic and $X \rightarrow Y$ any morphism. For every $E$ injective $I$, we have the induced commutative diagram of abelian groups

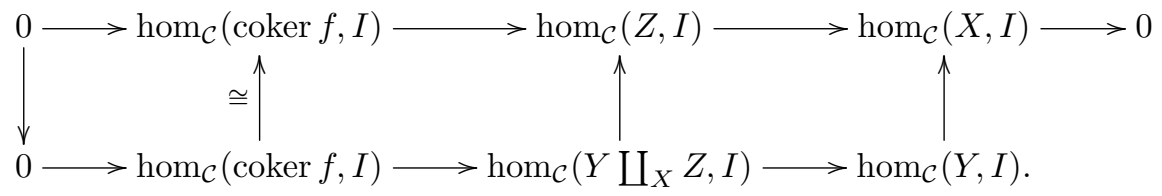

Exactness of the top row follows from $f$ being an $E$-monic together with $E$ having retractile monics, hence $E$ is its own retractile closure, hence (by the dual of Theorem 2.9) $E$-monics are precisely the maps which induce a surjection after applying $\operatorname{hom}_{\mathcal{C}}(-, I)$ for every $E$-injective $I$. The right-hand square in diagram (4) is a pullback square of abelian groups, by the universal property of the pushout. The top map in the square is a surjection, hence so is the bottom map, by Lemma 2.15. So $\operatorname{hom}_{\mathcal{C}}\left(Y \coprod_{X} Z, I\right) \rightarrow \operatorname{hom}_{\mathcal{C}}(Y, I)$ is a surjection for every $E$-injective $I$. Again since $E$ is its own retractile closure, this implies that $Y \rightarrow Y \coprod_{X} Z$ is an E-monic.

Lemma 2.17. Suppose $\mathcal{C}$ is an abelian category, $E$ an allowable class in $\mathcal{C}$ with retractile monics. Suppose $\mathcal{C}$ has enough $E$-injectives. Then a composite of E-monomorphisms is an E-monomorphism. 
Proof. Let $f: X \rightarrow Y$ and $g: Y \rightarrow Z$ be $E$-monomorphisms. Let $I$ be an $E$-injective object equipped with a map $X \rightarrow I$. Then, since $f$ is an $E$-monomorphism, $X \rightarrow I$ extends through $f$ to a map $Y \rightarrow I$, which in turn extends through $g$ since $g$ is an $E$-monomorphism. So every map to an $E$-injective from $Z$ extends through $g \circ f$. Now, by the dual of Heller's theorem 2.9, $g \circ f$ is an $E$-monomorphism.

Lemma 2.18. Let $\mathcal{C}$ be an abelian category and let $E$ be an allowable class in $\mathcal{C}$. Then a composite of two E-stable equivalences in $\mathcal{C}$ is an E-stable equivalence.

Proof. Let $X \stackrel{f}{\longrightarrow} Y \stackrel{g}{\longrightarrow} Z$ be a pair of $E$-stable equivalences. Then there exist $E$ projective objects $P_{X}, P_{Y}$ of $\mathcal{C}$ and morphisms

$$
\begin{aligned}
Y \stackrel{f^{\prime}}{\longrightarrow} X, & Z \stackrel{g^{\prime}}{\longrightarrow} Y, \\
X \stackrel{i_{X}}{\longrightarrow} P_{X}, & Y \stackrel{i_{Y}}{\longrightarrow} P_{Y}, \\
P_{X} \stackrel{s_{X}}{\longrightarrow} X, \text { and } & P_{Y} \stackrel{s_{Y}}{\longrightarrow} Y
\end{aligned}
$$

such that $f^{\prime} \circ f-\operatorname{id}_{X}=s_{X} \circ i_{X}$ and $g^{\prime} \circ g-\operatorname{id}_{Y}=s_{Y} \circ i_{Y}$. Then we have $f^{\prime} \circ g^{\prime} \circ$ $g \circ f-\mathrm{id}_{X}=s_{X} \circ i_{X}+f^{\prime} \circ s_{Y} \circ i_{Y} \circ f$, so $f^{\prime} \circ g^{\prime} \circ g \circ f-\mathrm{id}_{X}$ factors through the $E$-projective $P_{X} \oplus P_{Y}$. A similar argument applies to showing that $g \circ f \circ f^{\prime} \circ g^{\prime}-$ $\operatorname{id}_{Z}$ factors through an $E$-projective. So $g \circ f$ is an $E$-stable equivalence.

Definition-Proposition 2.19. Let $\mathcal{C}$ be an abelian category, let $E, F$ be allowable classes in $\mathcal{C}$ with $F \subseteq E$. Suppose each of the following conditions are satisfied:

- F has retractile monics.

- E has retractile monics and sectile epics.

- $\mathcal{C}$ has enough F-injectives.

- $\mathcal{C}$ has enough E-projectives and enough E-injectives.

- Every E-projective object is E-injective.

Then there exists a Waldhausen category structure on $\mathcal{C}$ in which the cofibrations are the F-monomorphisms and the weak equivalences are the E-stable equivalences. We write $\mathcal{C}_{E-\text { we }}^{F-c o f}$ for this Waldhausen category. This Waldhausen category satisfies the saturation axiom and the extension axiom.

Proof. We check the axioms from Definition 2.11. In the case of an abelian category $\mathcal{C}$ and allowable classes $E, F$ with the stated classes of cofibrations and weak equivalences, axioms (Cof 1 ) and (Cof 2) and (Weq 1) are immediate. That the class of cofibrations is closed under composition is Lemma 2.17. That the class of weak equivalences is closed under composition is Lemma 2.18. We show that the remaining axioms are satisfied:

- Axiom (Cof 3) is a consequence of Lemma 2.16.

- Axiom (Weq 2) is actually fairly substantial and takes some work to proveenough so that we moved this work into a paper of its own, [13]. In Corollary 4.4 of that paper, we prove that, if $E=F, \mathcal{C}$ has enough $E$-projectives and enough $E$-injectives, $E$ has sectile epics and retractile monics, and every $E$-projective object is $E$-injective, then $\mathcal{C}$ satisfies axiom (Weq 2). We refer the reader to 
that paper for the proof, which requires some work and a number of preliminary results, and would make the present paper much longer if we included it here. Once we have the result for $F=E$, it follows for $F \subseteq E$, since if diagram (1) has its horizontal maps $F$-monomorphisms, the horizontal maps are also then E-monomorphisms.

- The saturation axiom follows easily from Lemma 2.18 together with the observation that, if $X \stackrel{f}{\longrightarrow} Y$ is a $E$-stable equivalence and $Y \stackrel{f^{\prime}}{\longrightarrow} X$ is a morphism such that $f \circ f^{\prime}-\mathrm{id}_{Y}$ and $f^{\prime} \circ f-\mathrm{id}_{X}$ both factor through $E$-projective objects, then $f^{\prime}$ is also a $E$-stable equivalence. That is, $E$-stable equivalences have "upto-equivalence inverses."

- Finally, we handle the extension axiom. We begin by assuming that $E=F$, and that

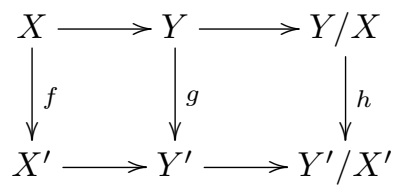

is a map of cofiber sequences (i.e., short exact sequences in $E$ ) and the maps $X \rightarrow X^{\prime}$ and $Y / X \rightarrow Y^{\prime} / X^{\prime}$ are $E$-stable equivalences. Then, for any object $M$ of $\mathcal{C}$, writing $H^{n}(-)$ for $\operatorname{Ext}_{\mathcal{C} / E}^{n}(-, M)$, we have the commutative diagram with exact rows

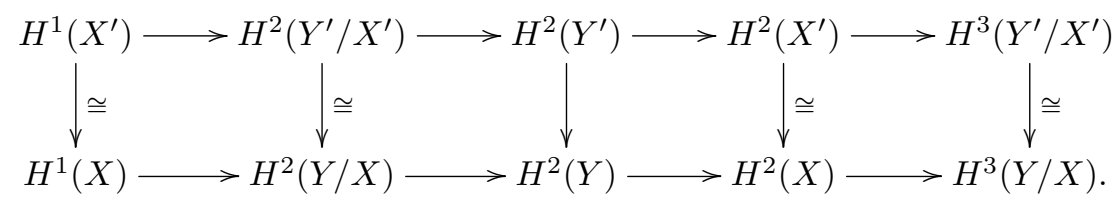

The vertical maps marked as isomorphisms are isomorphisms because an $E$-stable equivalence $A \longrightarrow B$ induces a natural equivalence of functors $\operatorname{Ext}_{\mathcal{C} / E}^{i}(B,-) \cong \operatorname{Ext}_{\mathcal{C} / E}^{i}(A,-)$ for all $i \geqslant 1$; this is Lemma 3.6 of [13]. By the Five Lemma, we then have a natural isomorphism of functors $\operatorname{Ext}_{\mathcal{C} / E}^{2}\left(Y^{\prime},-\right) \stackrel{\cong}{\longrightarrow}$ $\operatorname{Ext}_{\mathcal{C} / E}^{2}(Y,-)$. But since every $E$-projective is $E$-injective, this natural transformation being a natural isomorphism implies that $Y \rightarrow Y^{\prime}$ is an $E$-stable equivalence; this is Lemma 4.2 of [13]. Hence the extension axiom is satisfied if $E=F$. Now if we do not have $E=F$ but instead $F \subseteq E$, then the extension axiom remains satisfied, as we have fewer diagrams to check the extension axiom for.

\section{Absolute and relative quasi-Frobenius conditions}

\subsection{Definitions}

To our knowledge these definitions are all new. They are variants of the condition that every object embeds in a projective object, which Faith and Walker showed (see Theorem 1.1) to be equivalent, for categories of modules over a ring, to the ring being quasi-Frobenius. 
Definition 3.1. $\quad$ - Let $\mathcal{C}$ be an abelian category, $E, F$ a pair of allowable classes in $\mathcal{C}$. We say that $\mathcal{C}$ is cone-Frobenius relative to $E, F$ if, for any object $X$ of $\mathcal{C}$, there exists an $F$-monomorphism from $X$ to an $E$-projective object of $\mathcal{C}$.

We say that $\mathcal{C}$ is functorially cone-Frobenius relative to $E, F$ if there exists a functor $J: \mathcal{C} \rightarrow \mathcal{C}$ and a natural transformation $\eta: \operatorname{id}_{\mathcal{C}} \rightarrow J$ such that:

1. $J(X)$ is $E$-projective for every object $X$ of $\mathcal{C}$,

2. $\eta(X): X \rightarrow J(X)$ is an $F$-monomorphism for every object $X$ of $\mathcal{C}$,

3. if $f: X \rightarrow Y$ is an $F$-monomorphism then so is the map $J(f): J(X) \rightarrow$ $J(Y)$, and so is the universal map

$$
Y \coprod_{X} J(X) \rightarrow J(Y)
$$

4. and $J(0) \cong 0$.

We sometimes call the pair $J, \eta$ a cone functor on $\mathcal{C}$ relative to $E, F$. When $E, F$ are understood from context we simply call $J, \eta$ a relative cone functor.

- (The absolute case) If $E=F$ is the class of all short exact sequences in $\mathcal{C}$ and $\mathcal{C}$ is cone-Frobenius relative to $E, F$, then we say that $\mathcal{C}$ is cone-Frobenius. If $E=F$ is the class of all short exact sequences in $\mathcal{C}$ and $\mathcal{C}$ is functorially coneFrobenius relative to $E, F$, then we say that $\mathcal{C}$ is functorially cone-Frobenius.

The idea behind this definition is that the $F$-monomorphism and $E$-projective object together are a kind of "mapping cone," in the sense of homotopy theory, on $X$ : an embedding into a contractible object.

Under appropriate set-theoretic conditions on $\mathcal{C}$, the cone-Frobenius condition actually implies the functorial cone-Frobenius condition; this is the content of the appendix to this paper, section 5, especially Theorem 5.5 and Corollary 5.6. In particular, we prove that, for a quasi-Frobenius ring $R$, the category of left $R$-modules is functorially cone-Frobenius (Corollary 5.7), and for a quasi-Frobenius ring $R$ with only finitely many left ideals, the category of finitely generated left $R$-modules is functorially cone-Frobenius (Corollary 5.8). (The appendix does not logically depend on the rest of the paper.)

Now the Faith-Walker Theorem, stated above as Theorem 1.1, can be restated using our definitions: a ring $R$ is quasi-Frobenius if and only if the category of $R$ modules is cone-Frobenius.

\subsection{Existence of cylinder functors}

Now we show the equivalence of the functorial cone-Frobenius condition with the existence of cylinder functors satisfying the cylinder axiom. First, some lemmas:

Lemma 3.2. If $\mathcal{C}$ is an abelian category, $E$ an allowable class in $\mathcal{C}$ with sectile epics, and $\mathcal{C}$ has enough E-projectives, then a finite coproduct of members of $E$ is in $E$.

Proof. Let $I$ be a finite set and $0 \rightarrow X_{i} \rightarrow Y_{i} \rightarrow Z_{i} \rightarrow 0$ be a member of $E$ for every 
$i \in I$. Then, for any $E$-projective object $P$ of $\mathcal{C}$, we have the commutative diagram

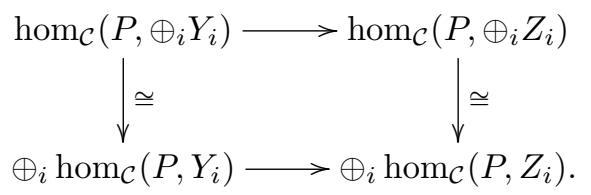

The bottom horizontal map is a surjection of abelian groups, so the top horizontal map is as well. Now by Heller's theorem 2.9, the map $\oplus_{i} Y_{i} \rightarrow \oplus_{i} Z_{i}$ is an E-epimorphism. So the short exact sequence $0 \rightarrow \oplus_{i} X_{i} \rightarrow \oplus_{i} Y_{i} \rightarrow \oplus_{i} Z_{i} \rightarrow 0$ is in $E$.

Lemma 3.3. (Shearing E-monics) Let $\mathcal{C}$ be an abelian category and let $E$ be an allowable class in $\mathcal{C}$. Suppose $X, Y, Z$ are objects in $\mathcal{C}$ and suppose we have $E$ monomorphisms $e: X \rightarrow Y$ and $f: Z \rightarrow Y$. Let $s$ be the morphism $s: X \oplus Z \rightarrow Y \oplus$ $Z$ given by the matrix of maps

$$
s=\left[\begin{array}{cc}
e & f \\
0 & \operatorname{id}_{Z}
\end{array}\right]
$$

Then coker $s$ is naturally isomorphic to coker e. Furthermore, if $\mathcal{C}$ has enough $E$ injectives and $E$ has retractile monics, then $s$ is an E-monomorphism.

Proof. That coker $e \cong$ coker $s$ follows immediately from the commutative diagram with exact rows and exact columns:

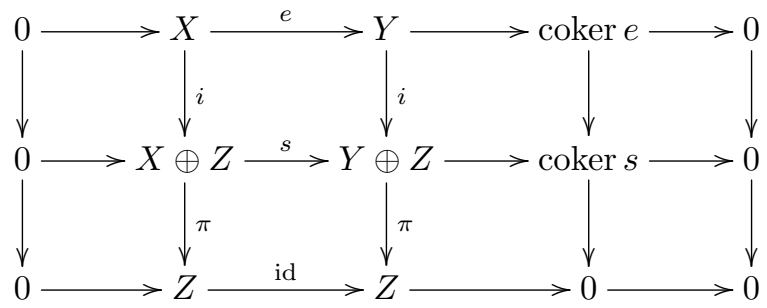

in which the maps marked $\pi$ are projections to the second summand, and the maps marked $i$ are inclusions as the first summand.

Now assume that $E$ has retractile monics, and let $t: Y \oplus Z \rightarrow Y \oplus Y$ be the map given by the matrix of maps

$$
t=\left[\begin{array}{cc}
\operatorname{id}_{Y} & -f \\
0 & f
\end{array}\right]
$$

Then a matrix multiplication reveals that the composite map $t \circ s: X \oplus Z \rightarrow Y \oplus Y$ is the direct sum map $e \oplus f$, a direct sum of $E$-monomorphisms, hence by Lemma 3.2, itself an $E$-monomorphism. (Note that, by taking the opposite category and noticing that the definition of an allowable class in an abelian category is self-dual, we get the conclusion of Lemma 3.2 if $E$ has retractile monics and $\mathcal{C}$ has enough $E$-injectives.) Now since $t \circ s$ is an $E$-monomorphism and $E$ is assumed to have retractile monics, $s$ is also an $E$-monomorphism.

Theorem 3.4. Let $\mathcal{C}, E, F$ be as in Proposition-Definition 2.19. Then the Waldhausen category $\mathcal{C}_{E-\text { we }}^{F-\text { cof }}$ admits a cylinder functor satisfying the cylinder axiom if and only if $\mathcal{C}$ is functorially cone-Frobenius relative to $E, F$. 
Proof. If $\mathcal{C}_{E-w e}^{F-c o f}$ has a cylinder functor satisfying the cylinder axiom, then the cylinder functor $I$ sends, for any object $X$ of $\mathcal{C}$, the map $X \rightarrow$ pt to the diagram

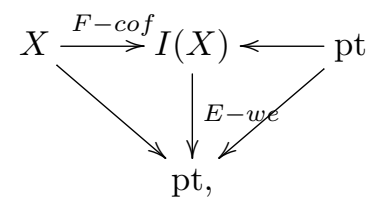

where the map marked $F-c o f$ is an $F$-monic and the map marked $E-w e$ is an $E$-stable equivalence. But for an object's map to the zero object to be an $E$-stable equivalence, this is equivalent to that object being $E$-projective. That $I(0)=0$ follows from condition (Cyl 2) in Definition 2.13, and if $f: X \rightarrow Y$ is an $F$-monomorphism, then so is $I(f)$ by (Cyl 1), and the universal map $Y \coprod_{X} I(X) \rightarrow I(Y)$ is also an $F$-monomorphism by applying ( $\mathrm{Cyl} 1)$ to the commutative square

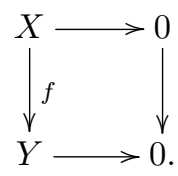

This completes one direction of the proof: $X \mapsto I(X)$ is a relative cone functor.

Now suppose we have a functor $J$ and natural transformation $e$ as in the definition of a relative cone functor in Definition 3.1. We claim that the functor sending any map $X \stackrel{f}{\longrightarrow} Y$ to the diagram

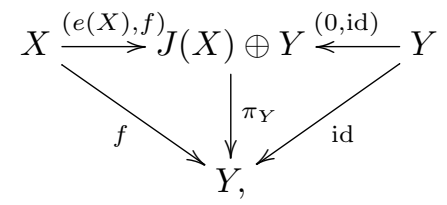

where $\pi_{Y}$ is projection to $Y$, is a cylinder functor on $\mathcal{C}$ satisfying the cylinder axiom. We check the conditions from Definition 2.13, with $T(f)$ defined to be $J(X) \oplus Y$ :

- We handle condition (Cyl 1) as follows: if $X^{\prime} \rightarrow X$ and $Y^{\prime} \rightarrow Y$ are $F$-monomorphisms fitting into a diagram as in (2), then the direct sum $T\left(f^{\prime}\right)=$ $J\left(X^{\prime}\right) \oplus Y^{\prime} \rightarrow J(X) \oplus Y=T(f)$ is an $F$-monomorphism by the assumption that $J$ sends $F$-monomorphisms to $F$-monomorphisms. The map

$$
X \coprod_{X^{\prime}}\left(J\left(X^{\prime}\right) \oplus Y^{\prime}\right) \coprod_{Y^{\prime}} Y=X \coprod_{X^{\prime}} T\left(f^{\prime}\right) \coprod_{Y^{\prime}} Y \rightarrow T(f)=J(X) \oplus Y
$$

of condition ( $\mathrm{Cyl} \mathrm{1)} \mathrm{is,} \mathrm{up} \mathrm{to} \mathrm{isomorphism,} \mathrm{the} \mathrm{direct} \mathrm{sum} \mathrm{of} \mathrm{map} \mathrm{(5)} \mathrm{with} \mathrm{the}$ identity map on $Y$. Consequently, map (3) is a cofibration if and only if map (5) is an $F$-monomorphism. The assumption that this latter condition holds, however, is part of the definition of the functorial cone-Frobenius condition. So the parts of condition ( $\mathrm{Cyl} 1$ ) which refer to cofibrations are satisfied.

Now suppose that $X^{\prime} \rightarrow X$ and $Y^{\prime} \rightarrow Y$ are $E$-stable equivalences. We also note that, since $J\left(X^{\prime}\right), J(X)$ are $E$-projective, the projections $J\left(X^{\prime}\right) \rightarrow$ pt and 
$J(X) \rightarrow$ pt are $E$-stable equivalences, and Lemma 2.18 gives us that the composite of either one with an $E$-stable inverse of the other is an $E$-stable equivalence between $J(X)$ and $J\left(X^{\prime}\right)$. So $T\left(f^{\prime}\right)=J\left(X^{\prime}\right) \oplus Y^{\prime} \rightarrow J(X) \oplus Y=T(f)$ is an $E$-stable equivalence.

So condition ( $\mathrm{Cyl} 1$ ) holds.

- Condition (Cyl 2) holds by the axiom $J(0) \cong 0$ in Definition 3.1.

- Lemma 3.3 implies that the direct sum map

$$
\left[\begin{array}{cc}
e(X) & 0 \\
f & \operatorname{id}_{Y}
\end{array}\right]: X \oplus Y \rightarrow J(X) \oplus Y
$$

is an $E$-monomorphism, i.e., a cofibration, so condition (Cyl 3) holds.

- The cylinder axiom follows from the projection $J(X) \oplus Y \rightarrow \operatorname{pt} \oplus Y \cong Y$ being a direct sum of $E$-stable equivalences, hence itself an $E$-stable equivalence.

Corollaries 3.5, 3.6, and 3.8 rely on a definition and a theorem from section 5 , the appendix to this paper. The appendix does not logically depend on anything else in the paper, so there is no danger of a circular argument.

Corollary 3.5. Let $\mathcal{C}$ be an abelian category, $E, F$ allowable classes in $\mathcal{C}$ satisfying the conditions:

- F has retractile monics.

- E has retractile monics and sectile epics.

- $\mathcal{C}$ has enough $F$-injectives.

- $\mathcal{C}$ has enough E-projectives and enough E-injectives.

- Every E-projective object is E-injective.

- There exists some cardinal number $\kappa$ such that $\mathcal{C}$ is $F$-monically $\kappa$-small and has all coproducts of cardinality $\leqslant \kappa$ (see Definition 5.1 for the definition of "F-monically $\kappa$-small").

- If $X, Y$ are objects of $\mathcal{C}$, then $\mathcal{C}$ has all products of cardinality less than or equal to that of the set $\operatorname{hom}_{\mathcal{C}}(X, Y)$.

- Every object of $\mathcal{C}$ embeds in an $F$-injective object by an $F$-monomorphism, i.e., for every object $X$ there exists an $F$-monomorphism from $X$ to an $F$-injective object.

- Every F-injective object of $\mathcal{C}$ is E-projective.

Then the category $\mathcal{C}$ admits a Waldhausen category structure in which the cofibrations are the F-monomorphisms, the weak equivalences are the E-stable equivalences, and this Waldhausen category satisfies the saturation and extension axioms and admits a cylinder functor satisfying the cylinder axiom.

Proof. Immediate from Definition-Proposition 2.19 and Theorems 3.4 and 5.5.

In the $E=F$ case:

Corollary 3.6. Let $\mathcal{C}$ be an abelian category, $E$ an allowable class in $\mathcal{C}$ satisfying the conditions: 
- E has retractile monics and sectile epics.

- $\mathcal{C}$ has enough E-projectives and enough E-injectives.

- An object is E-projective if and only if it is E-injective.

- There exists some cardinal number $\kappa$ such that $\mathcal{C}$ is E-monically $\kappa$-small and has all coproducts of cardinality $\leqslant \kappa$.

- If $X, Y$ are objects of $\mathcal{C}$, then $\mathcal{C}$ has all products of cardinality less than or equal to that of the set $\operatorname{hom}_{\mathcal{C}}(X, Y)$.

Then the category $\mathcal{C}$ admits a Waldhausen category structure in which the cofibrations are the E-monomorphisms, the weak equivalences are the E-stable equivalences, and this Waldhausen category satisfies the saturation and extension axioms and admits a cylinder functor satisfying the cylinder axiom.

Corollary 3.7. Suppose $\mathcal{C}$ is a quasi-Frobenius abelian category with enough projectives and functorially enough injectives. Then $\mathcal{C}$ admits the structure of a Waldhausen category in which the cofibrations are the monomorphisms and the weak equivalences are the stable equivalences. Furthermore, this Waldhausen category satisfies the saturation and extension axioms, and it admits a cylinder functor satisfying the cylinder axiom. We call this Waldhausen category the stable $G$-theory of $\mathcal{C}$, and we call its associated $K$-groups the stable $G$-theory groups of $\mathcal{C}$.

Corollary 3.8. Suppose $k$ is a finite field and $R$ a quasi-Frobenius $k$-algebra which is finite-dimensional as a $k$-vector space. Then the category of finitely generated (left) $R$-modules admits the structure of a Waldhausen category in which the cofibrations are the monomorphisms and the weak equivalences are the stable equivalences. Furthermore, this Waldhausen category satisfies the saturation and extension axioms, and it admits a cylinder functor satisfying the cylinder axiom.

\subsection{Multiplicative structure}

We recall Waldhausen's construction of a multiplicative structure on the $K$-theory of a Waldhausen category:

Theorem 3.9. (Waldhausen) Suppose $\mathcal{A}, \mathcal{B}, \mathcal{C}$ are Waldhausen categories and $F: \mathcal{A} \times \mathcal{B} \rightarrow \mathcal{C}$ is a functor satisfying each of the following conditions:

- If $M \rightarrow N$ is a cofibration in $\mathcal{A}$ and $L$ is an object of $\mathcal{B}$, then $F(M, L) \rightarrow$ $F(N, L)$ is a cofibration in $\mathcal{C}$.

- If $M \rightarrow N$ is a cofibration in $\mathcal{B}$ and $L$ is an object of $\mathcal{C}$, then $F(L, M) \rightarrow F(L, N)$ is a cofibration in $\mathcal{C}$.

- If $M^{\prime} \rightarrow M$ and $N^{\prime} \rightarrow N$ are cofibrations in $\mathcal{A}$ and $\mathcal{B}$, respectively, then the map $F\left(M^{\prime}, N\right) \coprod_{F\left(M^{\prime}, N^{\prime}\right)} F\left(M, N^{\prime}\right) \rightarrow F(M, N)$ is a cofibration in $\mathcal{C}$.

Then $F$ induces a natural pairing $\Omega|w S . \mathcal{A}| \times \Omega|w S . \mathcal{B}| \rightarrow \Omega \Omega|w S . S . \mathcal{C}| \stackrel{\cong}{\longrightarrow} \Omega|w S . \mathcal{C}|$.

Waldhausen's pairing is sufficiently natural to imply the following (see [11] for a good expository account of infinite loop spaces with ring structure, but note that the statement we can make, below, is about homotopy-commutative ring spaces and spectra, but not $E_{\infty}$-ring spaces or spectra): 
Corollary 3.10. Suppose $\mathcal{C}$ is a Waldhausen category which is equipped with a symmetric monoidal product $X, Y \mapsto X \otimes Y$ satisfying the conditions for the functor $F$ in Theorem 3.9. Then $\Omega|w S . \mathcal{C}|$ is a homotopy-commutative ring space, that is, the infinite loop space $\Omega|w S . \mathcal{C}|$ is equipped with a homotopy-commutative product that is compatible with its loop space structure.

Equivalently, when regarded as a spectrum using its infinite loop space structure, $\Omega|w S . \mathcal{C}|$ is a homotopy-commutative ring spectrum.

Lemma 3.11. Let $k$ be a field and let $M^{\prime}, M, N^{\prime}, N$ be finite-dimensional $k$-vector spaces. Suppose we have monomorphisms $M^{\prime} \rightarrow M$ and $N^{\prime} \rightarrow N$ of $k$-vector spaces. Then the canonical map $M^{\prime} \otimes_{k} N \coprod_{M^{\prime} \otimes_{k} N^{\prime}} M \otimes_{k} N^{\prime} \rightarrow M \otimes_{k} N$ is a monomorphism.

Proof. We will regard $M^{\prime}$ and $N^{\prime}$ as subspaces of $M$ and $N$, respectively. Choose a $k$-linear basis $\left\{m_{1}, \ldots, m_{i}\right\}$ for $M$ and a $k$-linear basis $\left\{n_{1}, \ldots, n_{j}\right\}$ for $N$ such that their restrictions to initial subsequences $\left\{m_{1}, \ldots, m_{i^{\prime}}\right\}$ and $\left\{n_{1}, \ldots, n_{j^{\prime}}\right\}$ form $k$-linear bases for $M^{\prime}$ and $N^{\prime}$, respectively. Then we have the short exact sequence of $k$-vector spaces

$$
0 \rightarrow M^{\prime} \otimes_{k} N^{\prime} \stackrel{f}{\longrightarrow} M^{\prime} \otimes_{k} N \oplus M \otimes_{k} N^{\prime} \rightarrow M^{\prime} \otimes_{k} N \coprod_{M^{\prime} \otimes_{k} N^{\prime}} M \otimes_{k} N^{\prime} \rightarrow 0 .
$$

We write $p$ for the composite map

$$
M^{\prime} \otimes_{k} N \oplus M \otimes_{k} N^{\prime} \rightarrow M^{\prime} \otimes_{k} N \coprod_{M^{\prime} \otimes_{k} N^{\prime}} M \otimes_{k} N^{\prime} \rightarrow M \otimes_{k} N .
$$

Suppose we have an element

$$
x=\left(\sum_{a=1}^{i^{\prime}} \sum_{b=1}^{j} \alpha_{a, b} m_{a} \otimes n_{b}, \sum_{a=1}^{i} \sum_{b=1}^{j^{\prime}} \beta_{a, b} m_{a} \otimes n_{b}\right) \in\left(M^{\prime} \otimes_{k} N\right) \oplus\left(M \otimes_{k} N^{\prime}\right)
$$

such that $p(x)=0$. Then we have:

$$
\begin{aligned}
0 & =\sum_{a=1}^{i^{\prime}} \sum_{b=1}^{j} \alpha_{a, b} m_{a} \otimes n_{b}-\sum_{a=1}^{i} \sum_{b=1}^{j^{\prime}} \beta_{a, b} m_{a} \otimes n_{b} \\
& =\sum_{a=1}^{i^{\prime}} \sum_{b=1}^{j^{\prime}}\left(\alpha_{a, b}-\beta_{a, b}\right) m_{a} \otimes n_{b}+\sum_{a=1}^{i^{\prime}} \sum_{b=j^{\prime}+1}^{j} \alpha_{a, b} m_{a} \otimes n_{b}+\sum_{a=i^{\prime}+1}^{i} \sum_{b=1}^{j^{\prime}}-\beta_{a, b} m_{a} \otimes n_{b},
\end{aligned}
$$

hence $\beta_{a, b}=0$ for all $a>i^{\prime}$, and $\alpha_{a, b}=0$ for all $b>j^{\prime}$, and $\alpha_{a, b}=\beta_{a, b}$ if $a \leqslant i^{\prime}$ and $b \leqslant j^{\prime}$. These conditions together imply that $x$ is in the subspace $M^{\prime} \otimes_{k} N^{\prime}$ of $\left(M^{\prime} \otimes_{k} N\right) \oplus\left(M \otimes_{k} N^{\prime}\right)$, hence the kernel of the composite map (6) is contained in $M^{\prime} \otimes_{k} N^{\prime}$, hence that the map from the quotient

$$
\left(\left(M^{\prime} \otimes_{k} N\right) \oplus\left(M \otimes_{k} N^{\prime}\right)\right) /\left(M^{\prime} \otimes_{k} N^{\prime}\right) \cong\left(M^{\prime} \otimes_{k} N\right) \coprod_{M^{\prime} \otimes_{k} N^{\prime}}\left(M \otimes_{k} N^{\prime}\right)
$$

to $M \otimes_{k} N$ is injective.

Proposition 3.12. Let $k$ be a field and let $A$ be a co-commutative Hopf algebra over $k$ which is finite-dimensional as a $k$-vector space. Let $\mathcal{G}_{\text {st }}(A)$ be the stable $G$-theory 
Waldhausen category of A from Corollary 3.7, that is, $\mathcal{G}_{\text {st }}(A)$ is the category of finitely generated (left) A-modules, with cofibrations the monomorphisms and weak equivalences the stable equivalences. Then the tensor product $\otimes_{k}$ over $k$ gives $\mathcal{G}_{\text {st }}(A)$ a symmetric monoidal product satisfying the assumptions of Corollary 3.10. Hence the stable $G$-theory space $\Omega\left|w S . \mathcal{G}_{\text {st }}\right|$ has a homotopy-commutative multiplication which distributes over the multiplication given by its infinite loop space structure; i.e., the stable $G$-theory spectrum of $\mathcal{G}_{s t}(A)$ is a homotopy-commutative ring spectrum.

Proof. Finite-dimensional Hopf algebras over fields are known to be quasi-Frobenius; see, e.g., [9] for this fact. That the tensor product over $k$ is symmetric monoidal follows from the Hopf algebra being co-commutative. That the tensor product over $k$ satisfies the first two conditions of Definition 3.9 is because every $A$-module is flat as a $k$ module, since $k$ is a field, so the tensor product over $k$ preserves monomorphisms. That the tensor product over $k$ satisfies the third condition is because of Lemma 3.11.

\section{Applications}

\subsection{The relationship between stable $G$-theory and other $G$-theories and $K$-theories}

We recall that stable $G$-theory was defined in Corollary 3.7. It sits naturally in a diagram relating it to algebraic $K$-theory, algebraic $G$-theory, and the "derived representation groups."

Now we define some notations we use to describe certain Waldhausen categories associated to an abelian category $\mathcal{C}$ :

Definition 4.1. Suppose $\mathcal{C}$ is an abelian category. We will write:

- $\mathcal{K}(\mathcal{C})$ for the $K$-theory category of $\mathcal{C}$, i.e., the Waldhausen category structure on the full subcategory generated by the projective objects of $\mathcal{C}$, where cofibrations are split inclusions and weak equivalences are isomorphisms.

- $\mathcal{G}^{\oplus}(\mathcal{C})$ for the split $G$-theory category of $\mathcal{C}$, i.e., the Waldhausen category structure on $\mathcal{C}$ where cofibrations are split inclusions and weak equivalences are isomorphisms.

- $\mathcal{G}(\mathcal{C})$ for the $G$-theory category of $\mathcal{C}$, i.e., the Waldhausen category structure on $\mathcal{C}$ where cofibrations are inclusions and weak equivalences are isomorphisms.

- When it exists: $\mathcal{G}_{s t}^{\oplus}(\mathcal{C})$ for the stable split $G$-theory category of $\mathcal{C}$, i.e., the Waldhausen category structure on $\mathcal{C}$ where cofibrations are split inclusions and weak equivalences are stable equivalences. (In the absolute sense, i.e., $E$-stable equivalences where $E$ is the allowable class of all short exact sequences in $\mathcal{C}$.)

- When it exists: $\mathcal{G}_{s t}(\mathcal{C})$ for the stable $G$-theory category of $\mathcal{C}$, i.e., the Waldhausen category structure on $\mathcal{C}$ where cofibrations are inclusions and weak equivalences are stable equivalences. (In the absolute sense, i.e., $E$-stable equivalences where $E$ is the allowable class of all short exact sequences in $\mathcal{C}$.)

Remark 4.2. We note that, if $R$ is a Noetherian ring and $\mathcal{C}$ is the category of finitely generated $R$-modules, then

$$
\pi_{n} \Omega|w S . \mathcal{K}(\mathcal{C})| \cong K_{n}(R)
$$

i.e., the Waldhausen $K$-theory of the $K$-theory Waldhausen category recovers the 
classical algebraic $K$-theory of $R$. Meanwhile,

$$
\pi_{n} \Omega|w S \cdot \mathcal{G}(\mathcal{C})| \cong G_{n}(R),
$$

i.e., the Waldhausen $G$-theory of the (nonsplit) $G$-theory Waldhausen category recovers the classical algebraic $G$-theory of $R$. (See section 1.9 of [14] for isomorphisms (7) and (8).)

The other theories are more obscure but still meaningful. In degree zero, $\pi_{0} \Omega\left|w S \cdot \mathcal{G}^{\oplus}(\mathcal{C})\right| \cong \operatorname{Rep}(R)$, the representation ring of $R$ - that is, the Grothendieck group completion of the semiring of isomorphism classes of finitely-generated $R$ modules. So we sometimes regard the split $G$-theory as the "derived representation theory" and the groups $\pi_{*} \Omega\left|w S \cdot \mathcal{G}^{\oplus}(\mathcal{C})\right|$ as the "derived representation groups" of $R$.

In degree zero, $\pi_{0} \Omega\left|w S . \mathcal{G}_{s t}^{\oplus}(\mathcal{C})\right| \cong \operatorname{StableRep}(R)$, the stable representation group of $R$ - that is, the Grothendieck group completion of the monoid of stable equivalence classes of finitely-generated $R$-modules. So we sometimes regard the split stable $G$ theory as the "derived stable representation theory" and the groups $\pi_{*} \Omega\left|w S . \mathcal{G}_{s t}^{\oplus}(\mathcal{C})\right|$ as the "derived stable representation groups" of $R$.

Finally, the results of this paper are really about the stable $G$-theory groups $\pi_{*} \Omega\left|w S . \mathcal{G}_{s t}(\mathcal{C})\right| \cong\left(G_{s t}\right)_{*}(\mathcal{C})$, as defined in Corollary 3.7. In degree zero, (nonsplit) stable $G$-theory is the stable representation group modulo the subgroup $A$ generated by all elements of the form $L-M+N$ where $0 \rightarrow L \rightarrow M \rightarrow N \rightarrow 0$ is a short exact sequence in $\mathcal{C}$.

Proposition 4.3. For any abelian category $\mathcal{C}$, we have a commutative diagram of topological spaces with nulhomotopic horizontal composites

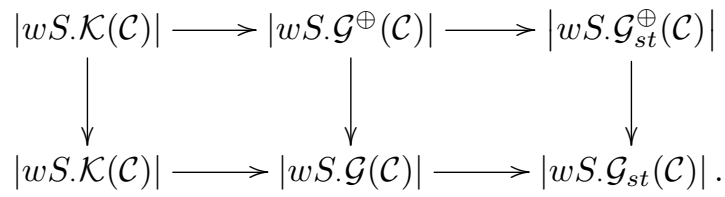

Suppose, furthermore, that $\mathcal{C}$ is functorially cone-Frobenius, has enough projectives, has enough injectives, and every projective object is injective. Then the bottom row in diagram (9) is a homotopy fiber sequence.

Proof. That the bottom row, under the stated assumptions, is a homotopy fiber sequence is an immediate consequence of Waldhausen's Fibration Theorem 2.14 and our Theorem 3.4. Everything else here is a consequence of elementary facts from [14].

\subsection{Applications to algebras}

In this section we will finally apply our results to actual rings and algebras! We will frequently assume that the rings in question are quasi-Frobenius. For an explanation of when and why this implies the functorial cone-Frobenius condition, see Proposition 5.2, Theorem 5.5, and Corollaries 5.6, 5.7, and 5.8. Throughout this section, whenever we assume that an algebra is over a finite field, the only reason we assume finiteness of the field is so that the above construction gives a cone functor on the finitely-generated module category; if one can extend this construction to finitelygenerated modules over algebras over more general fields, then one can do away with the finiteness assumption on the field. 
Proposition 4.4. (Stable $G$-theory is a delooping of relative algebraic $K$ theory) Suppose $k$ is a finite field and $A$ is a quasi-Frobenius $k$-algebra which is finite-dimensional as a $k$-vector space. Suppose $f: A \rightarrow k$ is a surjective morphism of $k$-algebras with nilpotent kernel. Write $K(A \rightarrow k)$ for the relative $K$-theory space of $f$, that is, the fiber of the map $\Omega|w S . \mathcal{K}(A)| \rightarrow \Omega|w S . \mathcal{K}(k)|$. Then we have a homotopy equivalence $K(A \rightarrow k) \simeq \Omega^{2}\left|w S . \mathcal{G}_{\text {st }}(A)\right|$, hence $\left(G_{s t}\right)_{n}(A) \cong K_{n-1}(A \rightarrow k)$ for all $n>0$.

Proof. We have the commutative diagram of (pointed) spaces

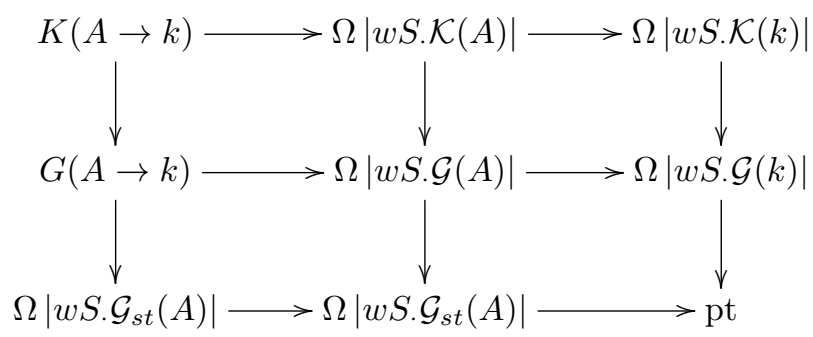

in which the spaces $K(A \rightarrow k)$ and $G(A \rightarrow k)$ are defined to be the homotopy fibers of the horizontal maps, so that the rows in diagram (10) are homotopy fiber sequences.

We note that the middle and right-hand columns in diagram (10) are also homotopy fiber sequences, by Proposition 4.3. Since all rows and the rightmost two columns in diagram (10) are homotopy fiber sequences, so is the left-hand column. By Quillen's filtration argument in $[\mathbf{1 2}]$, one also knows that any nilpotent extension of algebras over a field induces a homotopy equivalence in $G$-theory spaces, so the map $\Omega|w S . \mathcal{G}(A)| \rightarrow \Omega|w S . \mathcal{G}(k)|$ is a homotopy equivalence. So its fiber $G(A \rightarrow k)$ is contractible. Hence the left-hand column of diagram (10) reads, up to homotopy equivalence, $K(A \rightarrow k) \rightarrow \mathrm{pt} \rightarrow \Omega\left|w S . \mathcal{G}_{s t}(A)\right|$. Hence $K(A \rightarrow k) \simeq \Omega^{2}\left|w S . \mathcal{G}_{s t}(A)\right|$.

Now we recall Gabber's rigidity theorem, from, e.g., Chapter 4 of [15]:

Theorem 4.5. (Gabber) Suppose $A$ is a commutative ring and $(A, I)$ is a Hensel pair. Suppose $1 / i \in A$. Then, for all $n>0$, the $\operatorname{map} K_{n}(A ; \mathbb{Z} / i \mathbb{Z}) \rightarrow K_{n}(A / I ; \mathbb{Z} / i \mathbb{Z})$ is an isomorphism.

Theorem 4.6. Suppose $k$ is a finite field of characteristic $p$ and $A$ is a quasi-Frobenius local commutative $k$-algebra which is finite-dimensional as a $k$-vector space. Then we have the following computation of the stable $G$-theory groups of $A$ :

- $\left(G_{s t}\right)_{0}(A)$ is the abelian group whose objects are the stable equivalence classes of finitely-generated $A$-modules, modulo the relation $[L]+[N]=[M]$ if there exists a short exact sequence $0 \rightarrow L \rightarrow M \rightarrow N \rightarrow 0$.

- For $n>0,\left(G_{s t}\right)_{n}(A)$ is uniquely $\ell$-divisible for all primes $\ell \neq p$. $\left(S o\left(G_{s t}\right)_{n}(A)\right.$ has no summands of the form $\mathbb{Z} / \ell^{i} \mathbb{Z}$ or $\hat{\mathbb{Z}}_{\ell}$ or $(\mathbb{Q} / \mathbb{Z})_{\ell}$, but it may have summands of the form $\mathbb{Q}$, for example.)

- For $n>0$, the modulo $p^{i}$ stable $G$-theory $\left(G_{s t}\right)_{n}\left(A ; \mathbb{Z} / p^{i} \mathbb{Z}\right)$ is isomorphic to the topological cyclic homology $T C_{n-1}\left(A ; \mathbb{Z} / p^{i} \mathbb{Z}\right)$.

Proof. - The statement about $\left(G_{s t}\right)_{0}(A)$ is the usual identification of $K_{0}$ of a Waldhausen category; see Remark 4.2 . 
- Suppose $\ell$ is a prime distinct from $p$. From Gabber rigidity, Theorem 4.5, we know that $K_{n}\left(A \rightarrow k ; \mathbb{Z} / \ell^{i} \mathbb{Z}\right) \cong 0$ for $n \geqslant 1$. Since $A$ is assumed local, we also know that the map $K_{0}(A) \rightarrow K_{0}(k)$ is an isomorphism (the identity map from $\mathbb{Z}$ to $\mathbb{Z})$, so $K_{0}\left(A \rightarrow k ; \mathbb{Z} / \ell^{i} \mathbb{Z}\right)$ vanishes as well. From the short exact sequence

$$
0 \rightarrow K_{n}(A \rightarrow k) / \ell^{i} \rightarrow K_{n}\left(A \rightarrow k ; \mathbb{Z} / \ell^{i} \mathbb{Z}\right) \rightarrow \ell^{i} \text { torsion in } K_{n-1}(A \rightarrow k) \rightarrow 0
$$

and vanishing of the middle term for $n>0$, we then know that $K_{n}(A \rightarrow k) / \ell^{i} \cong$ 0 for $n>0$, and $K_{n}(A \rightarrow k)$ is $\ell^{i}$-torsion-free for $n \geqslant 0$. So, in particular, $K_{n}(A \rightarrow k)$ is uniquely $\ell$-divisible for all $n \geqslant 0$. By Proposition 4.4 , we know that $\left(G_{s t}\right)_{n}(A) \cong K_{n-1}(A \rightarrow k)$ for $n \geqslant 1$, so for $n \geqslant 1$ we conclude that $\left(G_{s t}\right)_{n}(A)$ is uniquely $\ell$-divisible.

- Since $A$ is finite, it is also Artinian, and so it is classical (see, e.g., Proposition 8.6 in [2]) that the maximal ideal of $A$ is nilpotent. So McCarthy's theorem (see, e.g., Madsen's article [8] for a good expository account) implies that $K_{n}(A \rightarrow$ $\left.k ; \mathbb{Z} / p^{i} \mathbb{Z}\right) \cong T C_{n}\left(A \rightarrow k ; \mathbb{Z} / p^{i} \mathbb{Z}\right)$. Now use Proposition 4.4.

One of our results (in Theorem 4.8, below) involves proving that certain stable $G$-theory spectra are complex-orientable. To prepare for that result, we provide the definition of a complex orientation.

Definition 4.7. Let $E$ be a homotopy-commutative ring spectrum. By a complex orientation on $E$ we mean a choice of element $\chi \in \tilde{E}^{2}(B U(1))$ with the property that $\chi$ maps to the multiplicative unit element $1 \in E^{0}(\mathrm{pt})$ under the composite

$$
\tilde{E}^{2}(B U(1)) \rightarrow \tilde{E}^{2}\left(S^{2}\right) \stackrel{\cong}{\longrightarrow} \tilde{E}^{0}\left(S^{0}\right) \stackrel{\cong}{\longrightarrow} E^{0}(\mathrm{pt}),
$$

where the left-hand map is the map induced in E-cohomology by inclusion of the 2-skeleton $S^{2}$ of $B U(1)$, the classifying space of complex line bundles.

If $E$ admits a complex orientation, we sometimes say that $E$ is complex-orientable.

Complex orientations are important for geometric purposes, since the element $\chi$ behaves essentially like a first Chern class, allowing one to carry out geometric arguments using $E$-cohomology that require characteristic classes for line bundles. Complex orientations also connect powerfully to number theory, via formal group laws: a complex orientation on a homotopy-commutative ring spectrum $E$ gives rise to a one-dimensional commutative formal group law on $\pi_{*}(E)$, and much of the homotopy theory of complex-orientable ring spectra can be described completely in terms of the moduli theory of one-dimensional formal groups. Adams's book [1] is an excellent reference for this material.

Now one wants to use Proposition 3.12 to get a multiplicative version of Theorem 4.6. So we have the following, which also uses Hesselholt-Madsen's computation of the topological cyclic homology of truncated polynomial rings (see, e.g., the survey article [8]; these computations of Hesselholt and Madsen are very substantial, far moreso than anything proven in the present paper!):

Theorem 4.8. Suppose $k$ is a finite field of characteristic p. Let $A \cong k[x] / x^{p^{n}}$ for some positive integer $n$. Then we have the following results:

- $\left(G_{s t}\right)_{0}(A) \cong \mathbb{Z} / p^{n} \mathbb{Z}$. 
- For all positive integers $m$,

$$
\left(G_{s t}\right)_{2 m}(A) \cong T C_{2 m-1}\left(A ; \hat{\mathbb{Z}}_{p}\right) \cong \mathbb{W}_{m p^{n}-1}(k) / V_{p^{n}} \mathbb{W}_{m-1}(k),
$$

where $\mathbb{W}_{i}(k)$ is the standard filtration quotient $i$ of the ring of big Witt vectors, that is, $\mathbb{W}_{i}(k)=(1+X k[[X]])^{\times} /\left(1+X^{i+1} k[[X]]\right)^{\times}$, and $V_{j}$ is the Verschiebung morphism sending a power series $f(X)$ to the power series $f\left(X^{j}\right)$.

- For all positive integers $m,\left(G_{s t}\right)_{2 m-1}(A) \cong 0$.

- $G_{s t}(A)$ is a complex-orientable ring spectrum.

Proof. - To get the isomorphism $\left(G_{s t}\right)_{0}(A) \cong \mathbb{Z} / p^{n} \mathbb{Z}$ we use the identification of degree zero stable $G$-theory, in Remark 4.2 , as the group of stable equivalence classes of finitely generated $A$-modules, modulo the relation splitting all short exact sequences. Now $A$ is a local Artinian principal ideal ring, hence its finitely-generated module category is Krull-Schmidt and every indecomposable finitely-generated module is cyclic, and for every $i \leqslant p^{n}$, we have the short exact sequence $0 \rightarrow k \rightarrow k[x] / x^{i} \rightarrow k[x] / x^{i-1} \rightarrow 0$ of $A$-modules, so we have the relation $[k]+\left[k[x] / x^{i-1}\right]=\left[k[x] / x^{i}\right]$ in $\left(G_{s t}\right)_{0}(A)$, so $[k]$ generates $\left(G_{s t}\right)_{0}(A)$. Finally, we observe that $\left[k[x] / x^{p^{n}}\right]=p^{n}[k]$ is zero in stable $G$-theory. So $\left(G_{s t}\right)_{0}(A) \cong \mathbb{Z} / p^{n} \mathbb{Z}$, generated by the stable equivalence class of the $A$ module $k$.

- In the proof of Proposition 4.4 we constructed a map of spectra $\Omega G_{s t}(A) \rightarrow$ $K(A \rightarrow k)$ which induces an isomorphism in $\pi_{i}$ for all $i>0$, and using McCarthy's theorem we saw that the cyclotomic trace map $K(A \rightarrow k) \wedge S / p^{j} \rightarrow$ $T C\left(A \rightarrow k ; \mathbb{Z} / p^{j} \mathbb{Z}\right)$ induces an isomorphism in $\pi_{i}$ for all $i>0$. The computation of $T C_{*}\left(A ; \hat{\mathbb{Z}}_{p}\right)$ is due to Hesselholt and Madsen, as in [8]: for $m>0$, we have

$$
T C_{2 m-1}\left(A ; \hat{\mathbb{Z}}_{p}\right) \cong T C_{2 m-1}\left(A \rightarrow k ; \hat{\mathbb{Z}}_{p}\right) \cong \mathbb{W}_{m p^{n}-1}(k) / V_{p^{n}} \mathbb{W}_{m-1}(k),
$$

and $T C\left(A ; \hat{\mathbb{Z}}_{p}\right)$ vanishes in positive even degrees.

Consequently, if $m>1$, then we have a commutative diagram with exact rows

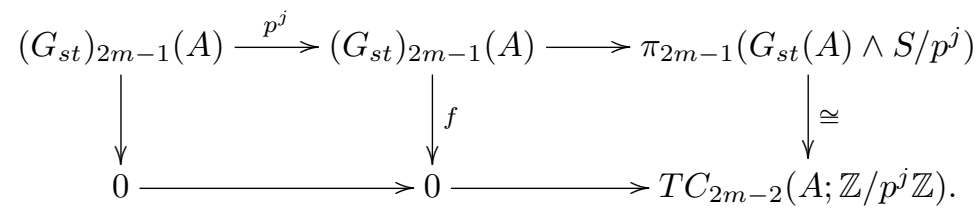

An easy diagram chase shows that, if $x \in\left(G_{s t}\right)_{2 m-1}(A)$ is in the kernel of $f$, then $x$ must be $p^{j}$-divisible. Since $\left(G_{s t}\right)_{2 m-1}(A)$ is a module over $\left(G_{s t}\right)_{0}(A) \cong \mathbb{Z} / p^{n} \mathbb{Z}$, if $j>n$ there can be no nonzero $p^{j}$-divisible elements in $\left(G_{s t}\right)_{2 m-1}(A)$. So $f$ is injective, so $\left(G_{s t}\right)_{2 m-1}(A) \cong 0$ for all $m>1$. When $m=1$ we get the same conclusion, that is, vanishing of $\left(G_{s t}\right)_{1}(A)$, from a diagram like (11) obtained from Theorem 4.6 and the vanishing of $K_{0}(A \rightarrow k)$. 
If $m \geqslant 1$, we also have a commutative diagram with exact rows

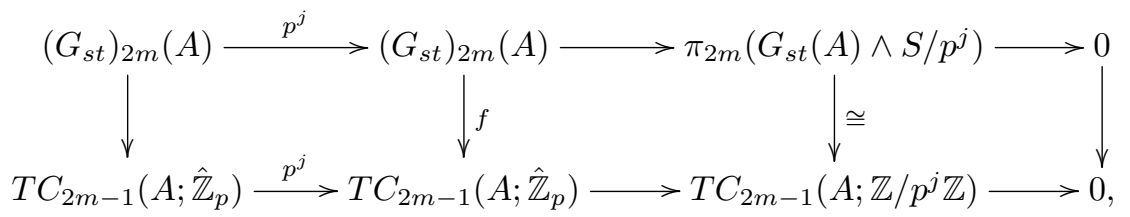

and a similar diagram chase and divisibility argument shows that $f$ is an isomorphism.

- We have the inclusion of the 2-skeleton $S^{2} \hookrightarrow B U(1)$ into the classifying space of complex line bundles, and the map it induces of $G_{s t}(A)$-cohomological AtiyahHirzebruch spectral sequences:

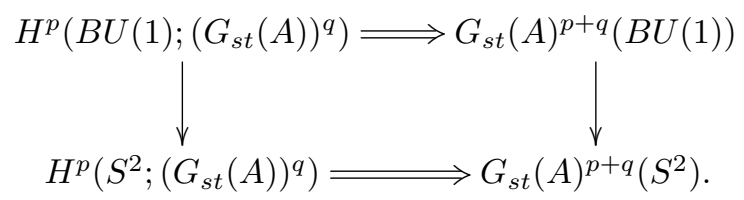

Since the homotopy groups of $G_{s t}(A)$ and the cohomology groups of $B U(1)$ are both concentrated in even degrees, there is no room for differentials in the spectral sequence for $B U(1)$. We conclude that the map of spectral sequences is simply projection on to the $p=0$ and $p=2$ columns, and that in particular, every element in $H^{2}\left(S^{2} ;\left(G_{s t}(A)\right)^{0}\right) \cong G_{s t}(A)^{2}\left(S^{2}\right)$ is in the image of the projection from $G_{s t}(A)^{2}(B U(1))$. So $G_{s t}(A)$ is complex-orientable.

\section{Appendix on functorial embeddings of modules into injectives}

This appendix tells us that, under appropriate conditions on an abelian category $\mathcal{C}$ with enough relative injectives, every object of $\mathcal{C}$ can be embedded functorially into a relative injective by a relative monomorphism. As a special case, we get a construction of a functorial embedding of any module into an injective module, and we show that this functorial embedding has certain nice properties which we use in the rest of the paper. This appendix does not logically depend on anything (except definitions) that appears elsewhere in the paper.

The functorial embeddings we construct (in the beginning of the proof of Theorem 5.5) are a generalization of a construction which appears in Bass's paper [3], and Bass writes there that he was told of it by Watts. I do not know if there is any earlier reference.

Definition 5.1. Let $\mathcal{C}$ be an abelian category, and let $E$ be an allowable class in $\mathcal{C}$. We will say that a set of objects $\left\{X_{s}\right\}_{s \in S}$ of $\mathcal{C}$ is an $E$-monic generating set for $\mathcal{C}$ if the following condition is satisfied:

- If $f: M \rightarrow N$ is a morphism in $\mathcal{C}$ such that $f \circ g$ is $E$-monic for every $E$ monomorphism $g: X_{s} \rightarrow M$, then $f$ is $E$-monic. 
If an $E$-monic generating set for $\mathcal{C}$ exists, then we say that $\mathcal{C}$ is $E$-monically small. If $\kappa$ is a cardinal number and an $E$-monic generating set for $\mathcal{C}$ exists with $\leqslant \kappa$ objects in it, then we say that $\mathcal{C}$ is $E$-monically $\kappa$-small.

For example:

Proposition 5.2. Let $R$ be a ring and let $\operatorname{Mod}(R)$ be the category of left $R$-modules. Let $E$ be the absolute allowable class on $\mathcal{C}$, i.e., $E$ is the class of all short exact sequences in $\mathcal{C}$. Let $\kappa$ be the (cardinal) number of left ideals of $R$. Then $\operatorname{Mod}(R)$ is $E$-monically $\kappa$-small. Furthermore, the category of finitely generated left $R$-modules is also E-monically $\kappa$-small.

Proof. Since $E$-monomorphisms for the absolute allowable class $E$ are simply monomorphisms, we drop the $E$ from the following proof. We claim that the set $\{R / J\}$, where $J$ ranges across all left ideals of $J$, is a monic generating set for $\operatorname{Mod}(R)$. Suppose $f: M \rightarrow N$ is a morphism in $\operatorname{Mod}(R)$, and choose a nonzero element $m \in M$. Then $m$ generates a cyclic submodule of $M$, i.e., we have a monomorphism of left $R$-modules

$$
R / J \rightarrow M
$$

sending 1 to $m$, for some left ideal $J$ of $R$. If every monomorphism $g: R / J \rightarrow M$ has the property that $f \circ g$ is monic, then monomorphism (12) has the property that its composite with $f$ is nonzero, and hence $f(m) \neq 0$. Hence $f(m) \neq 0$ for all $m$, hence $f$ is injective. So $\{R / J\}$ is a monic generating set for $\operatorname{Mod}(R)$.

The theorem we need for section 5 is Theorem 5.5 , but its proof requires a lemma:

Lemma 5.3. Let $\mathcal{C}$ be an abelian category, let $E$ be an allowable class in $\mathcal{C}$ with retractile monics, let $I: \mathcal{C} \rightarrow \mathcal{C}$ be a functor which sends the zero object to the zero object, and let $\eta: \operatorname{id}_{\mathcal{C}} \rightarrow I$ be a natural transformation with the property that $\eta(X): X \rightarrow I X$ is an E-monomorphism for all objects $X$ of $\mathcal{C}$. Suppose that $f: X \rightarrow Y$ is an $E$ monomorphism in $\mathcal{C}$, and suppose that If is also an E-monomorphism.

Then the canonical map

$$
Y \coprod_{X} I X \rightarrow I Y
$$

given by the universal property of the pushout, is also an E-monomorphism.

Proof. Let $p: Y \rightarrow$ coker $f$ be the projection-to-the-cokernel map. Since $I$ sends the zero object to the zero object, it also sends the zero map to the zero map, hence $I(p) \circ I(f)=I(p \circ f)=0$. Hence, by the universal property of the cokernel, the map $\eta(\operatorname{coker} f)$ : coker $f \rightarrow I$ (coker $f$ ) factors uniquely through the canonical map $e$ : coker $f \rightarrow \operatorname{coker} I f$. Since $\eta(\operatorname{coker} f)$ is $E$-monic by assumption, and since $E$ has retractile monics, $e$ is also $E$-monic.

Now $e$ along with the canonical comparison map $c: Y \coprod_{X} I X \rightarrow I Y$ of (13) both 
fit into the commutative diagram with exact rows

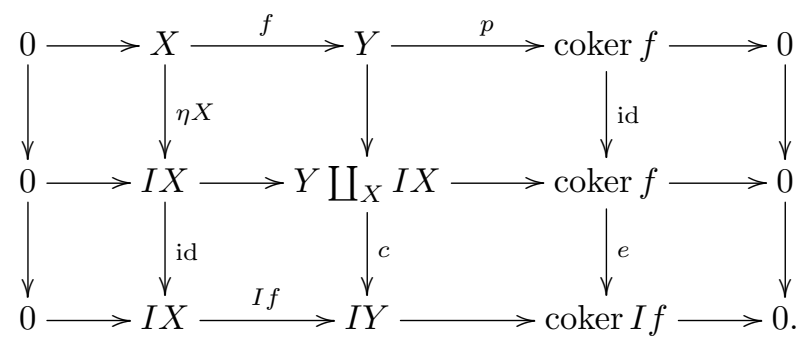

We already showed that $e$ is monic; it is then an extremely elementary exercise in homological algebra to show that $c$ is also monic. We still need to know that $c$ is $E$-monic; the proof is as follows. If we write $p r:$ coker $I f \rightarrow$ coker $e$ for the projection map to the cokernel, then the Snake Lemma implies that coker $e$ is also a cokernel for the map $c$, and the composite map $I Y \rightarrow$ coker If $\stackrel{p r}{\longrightarrow}$ coker $e$ is also a projectionto-the-cokernel map for $c$. Since we showed that $e$ is $E$-monic, we then know that the map $p r$ is $E$-epic, and since we assumed that $I f$ is $E$-monic, the projection map $I Y \rightarrow$ coker $I f$ is also $E$-epic. Consequently, the composite map $I Y \rightarrow$ coker $e$ is $E$ epic, and since that map is projection to a cokernel of $c$, the map $c$ is $E$-monic, as claimed.

Lemma 5.3 does not require the functor $I$ to be additive, and indeed, in the proof of Theorem 5.5, we apply Lemma 5.3 to a nonadditive functor (which, nevertheless, sends the zero object to the zero object).

Lemma 5.4. Let $\mathcal{C}$ be an abelian category, let $I$ be a set, and let $\left\{f_{i}: X_{i} \rightarrow Y_{i}\right\}_{i \in I}$ be a collection of split monomorphisms in $\mathcal{C}$. If the products $\prod_{i \in I} X_{i}$ and $\prod_{i \in I} Y_{i}$ exist in $\mathcal{C}$, then the product map $\prod_{i \in I} f_{i}: \prod_{i \in I} X_{i} \rightarrow \prod_{i \in I} Y_{i}$ is also a split monomorphism.

Proof. Choose a splitting map $s_{i}$ for each $f_{i}$. Then $\prod_{i \in I} s_{i}$ is a splitting map for $\prod_{i \in I} f_{i}$.

When we know that $\mathcal{C}$ is monically small relative to some allowable class, and that every object of $\mathcal{C}$ embeds into a relative injective, then in fact, we can functorially embed every object of $\mathcal{C}$ into a relative injective:

Theorem 5.5. Suppose $\mathcal{C}$ is an abelian category, E, $F$ allowable classes in $\mathcal{C}$. Suppose the following conditions are satisfied:

- F has retractile monics.

- $\mathcal{C}$ is F-monically $\kappa$-small.

- $\mathcal{C}$ has all coproducts of cardinality $\leqslant \kappa$.

- $\mathcal{C} h a s^{2}$

- all products, or

- all finite products and all hom-sets in $\mathcal{C}$ are finite, or

\footnotetext{
${ }^{2}$ The following conditions are simply those which guarantee that, if $X, Y$ are objects of $\mathcal{C}$, then $\mathcal{C}$ has all products of cardinality less than or equal to that of the set $\operatorname{hom}_{\mathcal{C}}(X, Y)$.
} 
- there exists an uncountable strongly inaccessible cardinal $\lambda$ which is a strict upper bound for the size of the hom-sets in $\mathcal{C}$, and $\mathcal{C}$ has all products of cardinality $<\lambda$ (this condition was suggested by the anonymous referee).

- Every object of $\mathcal{C}$ embeds in an $F$-injective object by an $F$-monomorphism, i.e., for every object $X$ there exists an $F$-monomorphism from $X$ to an $F$-injective object.

- Every F-injective object of $\mathcal{C}$ is E-projective.

Then $\mathcal{C}$ is functorially cone-Frobenius relative to $E, F$. (See Definition 3.1 for the definition of "functorially cone-Frobenius.")

Proof. First, choose an $F$-monic generating set $\left\{X_{s}\right\}_{s \in S}$ in $\mathcal{C}$, and choose an $F$ injective object $K$ of $\mathcal{C}$ and an $F$-monomorphism $\iota: \coprod_{s \in S} X_{s} \rightarrow K$.

Now let $I: \mathcal{C} \rightarrow \mathcal{C}$ be the functor given on objects by

$$
I(M)=\operatorname{ker}\left(\left(\prod_{f \in \operatorname{hom}_{\mathcal{C}}(M, K)} K\left\{e_{f}\right\}\right) \rightarrow K\left\{e_{0}\right\}\right),
$$

that is, $I(M)$ is the kernel of the projection-to-a-factor map $\prod_{f \in \operatorname{hom}_{\mathcal{C}}(M, K)} K\left\{e_{f}\right\} \rightarrow$ $K\left\{e_{0}\right\}$. (We need to define $I$ as the above kernel, and not merely as the product $\prod_{f \in \operatorname{hom}_{\mathcal{C}}(M, K)} K\left\{e_{f}\right\}$, in order for $I$ to send the zero object to the zero object, so that we can use Lemma 5.3 later in this same proof.) Here the symbols $e_{f}$ are formal symbols used to index the factors of $K$ in the product in (14). The functor $I$ is given on a morphism $\phi: M^{\prime} \rightarrow M$ in $\mathcal{C}$ by the map induced on kernels by

- the map given by the universal property of the product,

$$
\prod_{g \in \operatorname{hom}_{\mathcal{C}}\left(M^{\prime}, K\right)} K\left\{e_{g}\right\} \rightarrow \prod_{f \in \operatorname{hom}_{\mathcal{C}}(M, K)} K\left\{e_{f}\right\},
$$

- making use, for each $f: M \rightarrow K$ in $\mathcal{C}$, of the map

$$
\left(\prod_{g \in \operatorname{hom}_{\mathcal{C}}\left(M^{\prime}, K\right)} K\left\{e_{g}\right\}\right) \rightarrow K\left\{e_{f}\right\}
$$

given by the projection-to-a-factor map $\left(\prod_{g \in \operatorname{hom}_{\mathcal{C}}\left(M^{\prime}, K\right)} K\left\{e_{g}\right\}\right) \rightarrow K\left\{e_{f \circ \phi}\right\}$ followed by the identity map $K\left\{e_{f \circ \phi}\right\} \stackrel{\operatorname{id}_{K}}{\longrightarrow} K\left\{e_{f}\right\}$.

Now we define a natural transformation $\eta: \operatorname{id}_{\mathcal{C}} \rightarrow I$ as follows: for any object $X$ of $\mathcal{C}$, we can specify the map $\eta M: M \rightarrow I M$ by specifying its component mapping to $K\left\{e_{g}\right\}$ for each choice of $g$, by the universal property of the categorical product. We let the component mapping to $K\left\{e_{g}\right\}$ be simply $g$, that is, $g \in \operatorname{hom}_{\mathcal{C}}(M, K)$ is a map $M \rightarrow K$, and we let that map $g$ be the component map $M \rightarrow K\left\{e_{g}\right\}$. One checks easily that this construction is compatible with morphisms, hence defines a natural transformation.

For each object $X$ of $\mathcal{C}$, the object $I X$ is a product of $F$-injective objects, hence is $F$-injective, hence is $E$-projective, by assumption. The functor $I$ with the natural transformation $\eta: \operatorname{id}_{\mathcal{C}} \rightarrow I$ will be our relative cone functor; we will now check the axioms of Definition 3.1, in order. 
We first must check that $\eta M$ is actually an $F$-monomorphism. Here we use the assumption that $\left\{X_{s}\right\}_{s \in S}$ is an $F$-monic generating set. Choose some $s \in S$ and an $F$ monomorphism $\psi: X_{s} \rightarrow M$. (If no such $F$-monomorphism exists, then the conditions of Definition 5.1 are vacuously satisfied, and $\eta M$ is then automatically $F$-monic.) Let $i$ be the composite of the inclusion into the coproduct with the inclusion of the coproduct into the $F$-injective object $K: X_{s} \hookrightarrow \coprod_{s \in S} X_{s} \hookrightarrow K$, and observe that $i$ is a composite of a split monomorphism with an $F$-monomorphism, so $i$ is itself an $F$-monomorphism. So, in the commutative diagram

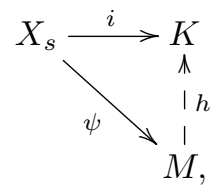

the dotted map marked $h$ exists by the universal property of an $F$-injective object. If $X_{s}$ is the zero object, then the composite map $X_{s} \stackrel{\psi}{\longrightarrow} M \stackrel{\eta M}{\longrightarrow} I M$ is automatically $F$ monic, as desired; so assume instead that $X_{s}$ is not the zero object. Then $i$ is not the zero map, so $h$ cannot be the zero map, so we have the projection $p r: I M \rightarrow K\left\{e_{h}\right\}$, and the composite map

$$
X_{s} \stackrel{\psi}{\longrightarrow} M \stackrel{\eta M}{\longrightarrow} I M \stackrel{p r}{\longrightarrow} K\left\{e_{h}\right\}
$$

is $h \circ \psi=i$, which is $F$-monic; since $F$ has retractile monics, $\eta M \circ \psi$ is also $F$-monic.

So, for every $F$-monomorphism $\psi: X_{s} \rightarrow M$, the composite $\eta M \circ \psi$ is $F$-monic. Now Definition 5.1 implies that $\eta M$ is $F$-monic, as desired.

Now we need to check that, if $\phi: M^{\prime} \rightarrow M$ is an $F$-monomorphism in $\mathcal{C}$, then so is the map $I \phi: I M^{\prime} \rightarrow I M$, and so is the universal map

$$
M^{\prime} \coprod_{M} I M \rightarrow I M^{\prime}
$$

Since $\phi$ is injective, the map $I \phi$ is a product of copies of the map $\operatorname{id}_{K}: K \rightarrow K$ and the zero map $0 \rightarrow K$, each of which are split monic. Now Lemma 5.4 implies that $I \phi$ is split monic, hence $F$-monic, as desired.

Now, since $I$ sends the zero object to the zero object, Lemma 5.3 also implies that the map (15) is $F$-monic.

As a special case:

Corollary 5.6. Suppose $\mathcal{C}$ is an abelian category with enough injectives, with all small products and coproducts, and which is monically small. Suppose that every injective object in $\mathcal{C}$ is projective. Then $\mathcal{C}$ is functorially cone-Frobenius.

As an even more special case:

Corollary 5.7. Let $R$ be a quasi-Frobenius ring. Then the category of left $R$-modules is functorially cone-Frobenius.

Finally, there is the question of when we have the functorial cone-Frobenius condition on the finitely generated modules over a ring: 
Corollary 5.8. Let $R$ be an quasi-Frobenius ring which is finite, i.e., $R$ has only finitely many elements. Then the category of finitely generated left $R$-modules is functorially cone-Frobenius.

Proof. We need to check that the assumptions in the statement of Theorem 5.5 are satisfied. Since $R$ is finite, $R$ has only finitely many ideals, so by Proposition 5.2 , the category of finitely generated left $R$-modules is $\kappa$-small for some finite cardinal $\kappa$. Of course then coproducts of cardinality $\leqslant \kappa$ exist in the category of finitely generated left $R$-modules. Since $R$ is finite, there are only finitely many homomorphisms from any finitely generated left $R$-module to any other finitely generated left $R$-module, so the category of finitely generated left $R$-modules has all products indexed by its hom-sets.

The last condition to check is that every finitely generated left $R$-module embeds in a projective finitely generated left $R$-module. Since $R$ is quasi-Frobenius, every finitely generated left $R$-module $M$ embeds in a free left $R$-module, that is, $\coprod_{i \in I} R\left\{e_{i}\right\}$ for some set $I$, where the symbols $e_{i}$ are formal symbols used to keep track of the copies of $R$. Let $\left\{g_{1}, \ldots, g_{n}\right\}$ be a set of generators for $M$. The image of each $g_{j}$ in $\coprod_{i \in I} R\left\{e_{i}\right\}$ is of the form $\sum_{i \in I} r_{i, j} e_{i}$, with each $r_{i, j}$ and, for each $j$, all but finitely many $r_{i, j}$ are zero. Since there are only finitely many $g_{j}$, the image of each $g_{1}, \ldots, g_{n}$ is contained in the sub- $R$-module of $\coprod_{i \in I} R\left\{e_{i}\right\}$ generated by a finite subset of the generators $e_{i}$. Hence $M$ embeds in a finitely generated free left $R$-module.

So the functorial cone-Frobenius condition seems to be a reasonable and oftensatisfied one.

\section{References}

[1] J.F. Adams. Stable Homotopy and Generalised Homology. Chicago Lectures in Mathematics. University of Chicago Press, Chicago, IL, 1995. Reprint of the 1974 original.

[2] M.F. Atiyah and I.G. Macdonald. Introduction to Commutative Algebra. Addison-Wesley Publishing Co., Reading, Mass.-London-Don Mills, Ont., 1969.

[3] H. Bass. Injective dimension in Noetherian rings. Trans. Amer. Math. Soc., 102:18-29, 1962.

[4] C. Faith. Embedding modules in projectives: A report on a problem. In Advances in Non-Commutative Ring Theory, volume 951 of Lecture Notes in Mathematics, pages 21-40, 1982.

[5] G. Garkusha. On the homotopy cofibre spectrum of $K(R) \rightarrow G(R)$. J. Algebra Appl., 1(3):327-334, 2002.

[6] M. Hovey. Model Categories, volume 63 of Mathematical Surveys and Monographs. American Mathematical Society, Providence, RI, 1999.

[7] S. Mac Lane. Homology. Classics in Mathematics. Springer-Verlag, Berlin, 1995. Reprint of the 1975 edition.

[8] I. Madsen. Algebraic K-theory and traces. In Current Developments in Mathematics, 1995 (Cambridge, MA), pages 191-321. Int. Press, Cambridge, MA, 1994. 
[9] H.R. Margolis. Spectra and the Steenrod Algebra, volume 29 of North-Holland Mathematical Library. North-Holland Publishing Co., Amsterdam, 1983.

[10] J.P. May. Infinite loop space theory. Bull. Amer. Math. Soc., 83(4):456-494, 1977.

[11] J.P. May. What precisely are $E_{\infty}$ ring spaces and $E_{\infty}$ ring spectra? In New Topological Contexts for Galois Theory and Algebraic Geometry (BIRS 2008), volume 16 of Geometry $\&$ Topology Monograph, pages 215-282. Geom. Topol. Publ., Coventry, 2009.

[12] D. Quillen. Higher algebraic K-theory. I. In Algebraic K-Theory, I: Higher K-Theories (Proc. Conf., Battelle Memorial Inst., Seattle, Wash., 1972), volume 341 of Lecture Notes in Mathematics, pages 85-147. Springer, Berlin, 1973.

[13] A. Salch. Homotopy colimits in stable representation theory. Homology Homotopy Appl., 15:331-360, 2013.

[14] F. Waldhausen. Algebraic $K$-theory of spaces. In Algebraic and Geometric Topology (New Brunswick, N.J., 1983), volume 1126 of Lecture Notes in Mathematics, pages 318-419. Springer, Berlin, 1985.

[15] C.A. Weibel. The K-Book, volume 145 of Graduate Studies in Mathematics. American Mathematical Society, Providence, RI, 2013. An introduction to algebraic $K$-theory.

Andrew Salch asalch@math.wayne.edu

Department of Mathematics, Wayne State University, 1150 F/AB, 656 W. Kirby, Detroit, MI, 48202, USA 